\title{
Tutorial
}

\section{Merge of high harmonic generation from gases and solids and its implications for attosecond science}

\author{
G Vampa ${ }^{1}$ and $\mathrm{T}$ Brabec \\ Department of Physics, University of Ottawa, Ottawa, ON K1N 6N5, Canada \\ E-mail: gvampa@stanford.edu
}

Received 12 August 2015, revised 28 November 2016

Accepted for publication 8 December 2016

Published 23 March 2017

\begin{abstract}
High harmonic generation (HHG) in atomic and molecular gases builds the foundation of attosecond science. In recent experiments HHG has been demonstrated in solids for the first time. A theoretical analysis has revealed that one of the mechanisms driving HHG in semiconductors is similar to the one in atomic and molecular gases. As a result, many of the processes developed for attosecond science in gases can be adapted and applied to the condensed matter phase. In this tutorial, the connection between atomic and solid HHG is summarized with covering both theoretical and experimental work, and some implications for attosecond science in solids are presented.
\end{abstract}

Keywords: high harmonic generation, solids, nonlinear optics

(Some figures may appear in colour only in the online journal)

\section{Introduction}

High harmonic generation (HHG) is an extremely nonlinear optical process that converts tens or hundreds (sometimes even thousands) of low-energy photons into one soft x-ray photon. Traditionally, the mediators of the photon exchange are isolated atoms (rare gases especially) or small molecules. However, the process cannot be understood in terms of a perturbation expansion of the polarization of the atom or molecule as is done in conventional nonlinear optics [1].

To initiate the process, the least bound electrons must tunnel to the vacuum under the Coulomb barrier of the atom or molecule that is lowered when the intense laser field is close to maximum. Once the electron tunnelled, it is accelerated on a recolliding path with the ion. Upon collision, the electron can recombine with the ion and release the kinetic energy gained in the laser field as one high-energy photon-a high harmonic photon. The whole process is coherently

\footnotetext{
1 Present address: Stanford PULSE Institute, SLAC National Accelerator Laboratory, Menlo Park, CA 94025, United States of America.
}

driven by the oscillations of the laser electric field and it lasts only a fraction of the optical cycle.

For the electron to efficiently tunnel and to gain enough momentum, the laser field must be strong and the laser photon energy must be much smaller than the ionization potential of the atom or molecule. How strong should the laser field be? Considering that the ionization potential is on the order of $10 \mathrm{eV}$ and the atomic radius on the order of $1 \AA$, the required field is about $10 \mathrm{~V}^{-1}$, equivalent to a peak intensity of $10^{15}$ $\mathrm{W} \mathrm{cm}{ }^{-2}$. Most commonly used laser sources have been amplified femtosecond Ti:Sapphire lasers, but in recent years new sources at longer wavelengths have been developed as well.

The spectacular coherence properties of HHG have been used to image molecular orbitals [2,3], to map the evolution of chemical reactions [4], to study multi-electron dynamics in molecules [5,6] and to synthesize light flashes that last only some tens of attoseconds ( 1 as $\left.=10^{-18} \mathrm{~s}\right)$ [7]. These pulses are used to perform pump-probe studies with attosecond resolution in gases [8, 9], solids [10-12] and biomolecules [13]. 
Can the broad success of attosecond and high harmonic spectroscopies in gases be extended to solids? The answer is possibly yes, thanks to a breakthrough that occurred in 2011 , when Ghimire and co-workers [14] reported the observation of HHG from the bulk of a $\mathrm{ZnO}$ crystal, a semiconductor with a direct band-gap of $3.2 \mathrm{eV}$. Since then, high harmonics have also been obtained from a dielectric [15] and smaller-gap semiconductors $[16,17]$. It is now clear that the process can occur in a wide range of materials, with direct and indirect band-gaps alike. All these demonstrations were possible thanks to the latest advances in laser technology: in [15], the authors used half-cycle pulses from a Ti:Sapphire laser to generate high harmonics from $\mathrm{SiO}_{2}$, a $9 \mathrm{eV}$ band-gap solid; in [16], the authors used a highly intense terahertz source; and in [14] the authors used a femtosecond mid-infrared laser with a central wavelength $>3 \mu \mathrm{m}$.

Attosecond science in atomic and molecular gases has matured into a highly successful research field. To establish similarly successful attosecond science in solids, and to extend it to technology, however, the underlying generating mechanisms must be understood. This topic has been considerably debated. Among the possible sources of high harmonics, we have identified one that is equivalent to the recollision mechanism of atomic $\mathrm{HHG}$, and that dominates the emission for a range of materials and wavelengths. This similarity builds the basis to adapt and apply attosecond technology developed for atomic and molecular gases to the condensed matter phase.

This tutorial reviews the physics underlying HHG from bulk crystals, with particular emphasis on the recollision mechanism and the similarities and differences with the atomic case. We also briefly touch upon what we have learned about the competing mechanisms, but we refer the reader to other literature cited in the text for more exhaustive explanations on this matter.

The tutorial is organized as follows: in section 2 we present the semiclassical model of recollision-based HHG for atoms and solids and highlight the main similarities and differences between the two. In section 3, the semiclassical model for solids is justified based on saddle point integration of the Schrödinger equation, along with a discussion of the competing mechanisms and their relative importance. In section 4, we present an experimental evidence for recolliding electron-hole pairs and analyse some predictions of the model. At the end, we draw the conclusions and highlight open questions.

\section{Semiclassical model}

To understand the basics of HHG in bulk solids it is most useful to describe a semiclassical model of the motion of electron-hole pairs that are created by the laser field. In this section, the model is introduced by a heuristic extension of the more familiar semiclassical model of HHG from atoms ([18] includes a more comprehensive review of atomic HHG). The spirit of this section is to answer the question: 'How would a semiclassical model of high harmonics from solids be, had the generating mechanism been equivalent to the atomic case?' To answer this question, we first review the atomic case, then transition from atoms to solids. Justification of the model through saddle point integration of the Schrödinger equation is deferred to section 3 .

\subsection{The atomic case}

The basic features of the HHG process in gases can be grasped with a semiclassical three-step model [19]. In the first step, an electron, initially bound to an atomic core, tunnels through the potential barrier lowered by the strong laser field and is freed with almost zero initial kinetic energy, leaving a hole strongly localized on the atom. In the second step, the electron is accelerated away by the strong laser field and, if the time at which the electron is ionized relative to the peak of the laser electric field is right, it can return towards the ionic core. In the third step, the electron meets the hole and can recombine with it, emitting the kinetic energy that it picked up in the laser field plus the ionization potential of the atom as a high harmonic photon.

2.1.1. The ionization step. For the purpose of $\mathrm{HHG}$, the major role played by the first step is to free the electron from the atom. Although the electron crossing the barrier has some influence over the high harmonics [20], in this context the nature of the ionization step is disregarded. The quantum nature of ionization leaves uncertainty in the time at which the electron crosses the barrier and begins to accelerate away from the hole. It is this uncertainty that makes the model 'semiclassical'. Here we neglect the uncertainty and focus on the classical part of the process.

2.1.2. The propagation step. The analysis starts with a free electron subject to the laser electric field $\mathbf{F}(t)=F_{0} \cos \left(\omega_{0} t\right) \hat{\mathbf{x}}$. In atomic units $\left(e=m=\hbar=4 \pi \epsilon_{0}=1\right)$, the momentum of the electron is:

$$
\begin{aligned}
\mathbf{k} & \equiv \dot{\mathbf{x}}(t)=-\hat{\mathbf{x}} \int_{t^{\prime}}^{t} F_{0} \cos \left(\omega_{0} \tau\right) \mathrm{d} \tau+\mathbf{v}_{0} \\
& =-\frac{F_{0}}{\omega_{0}}\left[\sin \left(\omega_{0} t\right)-\sin \left(\omega_{0} t^{\prime}\right)\right] \hat{\mathbf{x}}+\mathbf{v}_{0} .
\end{aligned}
$$

In the following, the vector notation is dropped because the motion is confined along the (linear) laser polarization. The quantum model suggests that the electron tunnels with approximately zero initial velocity: $\mathbf{v}_{0} \simeq 0$. The time the electron begins to move, $t^{\prime}$, is measured relative to the peak of the laser field at $t^{\prime}=0$. The position of the electron is:

$$
\begin{aligned}
x(t)= & \int_{t^{\prime}}^{t} \dot{x}(\tau) \mathrm{d} \tau=\frac{F_{0}}{\omega_{0}^{2}}\left[\cos \left(\omega_{0} t\right)-\cos \left(\omega_{0} t^{\prime}\right)\right. \\
& \left.+\sin \left(\omega_{0} t^{\prime}\right)\left(t-t^{\prime}\right)\right] .
\end{aligned}
$$

When $t^{\prime} \neq 0$ the electron oscillates with non-zero average momentum $k_{\text {drift }} \propto \sin \left(\omega_{0} t^{\prime}\right)$, which drifts its mean position relative to the hole (fixed at $x=0$ ) linearly in time. The later the time of ionization, the stronger the electron drifts (see figure 1(a)). An electron born at the peak of the field re-encounters the hole after each laser cycle, while one born after 

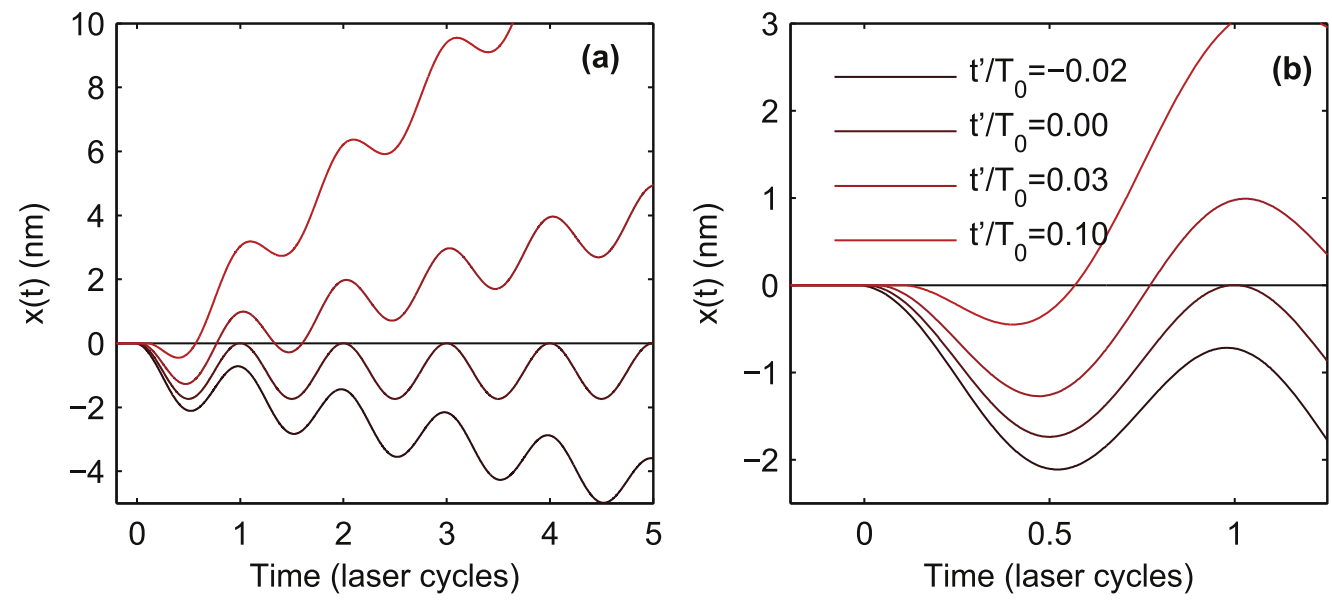

Figure 1. Trajectories of electrons ionized at different times relative to the peak of the electric field, set at an intensity of $10^{14} \mathrm{~W} \mathrm{~cm}{ }^{-2}$ with central wavelength of $800 \mathrm{~nm}$. (b) Is a close up within one laser cycle. $T_{0}$ is the laser cycle.

the peak re-encounters it a limited number of times, and earlier in the cycle (see figure 1(b)). Another consequence of the classical motion is that, if the Coulomb attraction of the ion is neglected, an electron born before the peak of the field never reencounters the hole, because the drift velocity changes sign and acts together with the electric field right after ionization to accelerate the electron farther.

The most common experiments focus femtosecond laser pulses with a bandwidth centred about $800 \mathrm{~nm}$ to intensities about $10^{14} \mathrm{~W} \mathrm{~cm}^{-2}$. In these conditions the maximum excursion of the electron before recollision $\left(2 F_{0} / \omega_{0}^{2}\right.$, from equation (2)) is on the order of $1 \mathrm{~nm}$, therefore much larger than the atomic radius $(\sim 0.1 \mathrm{~nm})$. The cycle-averaged kinetic energy of electrons born at the peak of the field, the ponderomotive energy $U_{p}$, is typically used as a good metric of the energetics of the process. From equation (1) it is easily derived that $U_{p}=F_{0}^{2} /\left(2 \omega_{0}\right)^{2} \simeq 10 \mathrm{eV}$. Ponderomotive energies as high as $1000 \mathrm{eV}$ can be reached in experiments with laser fields of longer wavelength and intensities in the $10^{15} \mathrm{~W} \mathrm{~cm}^{-2}$ range [21].

2.1.3. Recollision and emission of high harmonics. Figure 1(b) shows the link between the times of creation and of recollision of the electron-hole pair: the later the electron is ionized, the sooner it recollides with the hole (fixed at $x=0$ ). The kinetic energy $T$ of the electron is transferred to a high harmonic photon upon recombination of the electron with its associated hole. Its energy is:

$$
\hbar \omega=T+I_{p},
$$

where $I_{p}$ is the ionization potential of the atom. The electron's velocity at the time of recollision, the slope of the lines at the zero crossings, also depends on the time of ionization. This is shown in figure 2 . It is found numerically that the harmonic photon energy peaks for an ionization phase of $\sim 0.31 \mathrm{rad}$ at the energy given by $[18,19]$ :

$$
\hbar \omega_{c}=3.17 U_{p}+I_{p} .
$$

The existence of electron trajectories completely determined by any variable among time of ionization, time of recollision and

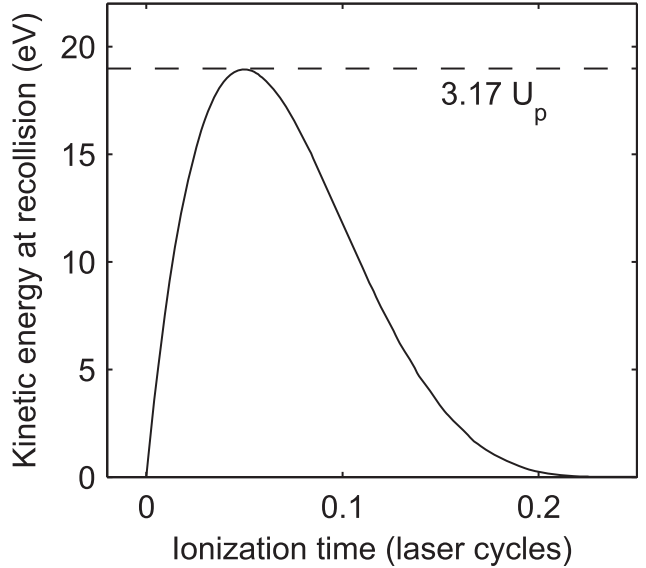

Figure 2. Kinetic energy of the electron at the time of recollision, as a function of the time of ionization. The maximum kinetic energy is determined by $3.17 U_{p}$. The laser intensity is $10^{14} \mathrm{~W} \mathrm{~cm}^{-2}$, central wavelength is $800 \mathrm{~nm}$.

emitted photon energy, is perhaps the most peculiar aspect of atomic HHG.

The bandwidth of the emitted radiation, shown in figure 2 , is continuous. It does not explain the generation of only the odd-order harmonics, observed in experiments ${ }^{2}$ [18]. Odd harmonics result from the interference between emissions at each laser half-cycle ${ }^{3}$. Harmonic radiation emitted at successive half-cycles has opposite phase, due to the electron approaching the ion from opposite sides. The phase flip results in constructive interference of harmonic frequencies that are exactly odd multiples of the driving laser frequency. This is demonstrated below by summing only two harmonic waves of frequency $n \omega_{0}$ emitted with a delay of half of the

2 A continuous bandwidth is experimentally observed under special circumstances.

3 Interference arises in the act of measuring the spectrum. Sufficient spectral resolution comes to the price of lengthening the harmonic pulses by more than their temporal separation, which allows them to interfere. 


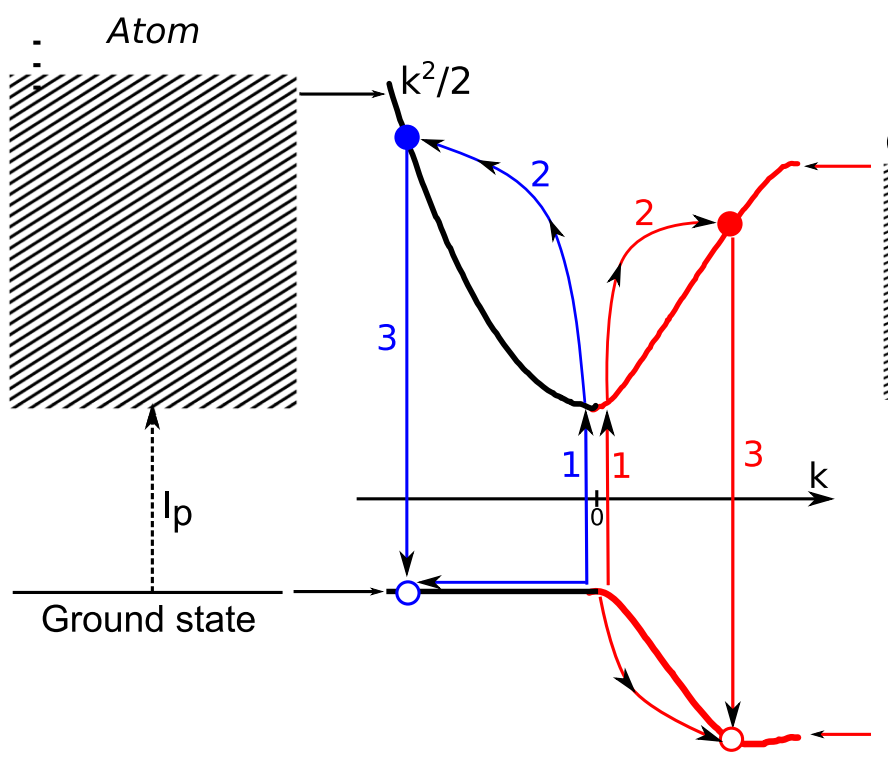

Solid

Conduction band
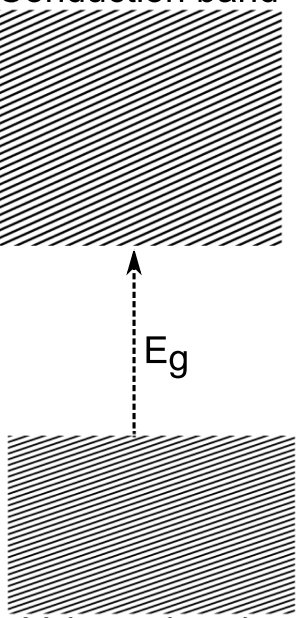

Valence band

Figure 3. Energy diagrams of atoms and solids. In an atom (left), the ground state is a localized energy state, separated by the ionization potential $I_{p}$ from the continuum states (band) of vacuum (the dashed square). In momentum space, the latter is a parabola (black line in the middle). In a solid (right), both ground and excited states form bands (valence and conduction, respectively), separated by the minimum energy gap $E_{g}$. They are more complicated functions of momentum (red lines in the middle). Here, the valence and first conduction band of a $\mathrm{ZnO}$ crystal are shown. They are calculated with density functional theory (implemented by the ABINIT code [22]). High harmonic generation proceeds through creation of an electron-hole pair (labelled ' 1 ', blue colours for the atomic case, red for the solid), acceleration of the pair to high momentum (labelled ' 2 '), and recombination of the electron with the hole (labelled ' 3 '). Although in the atomic case the pair is created with zero initial momentum $(k=0)$, in a solid this is not necessarily the case.

fundamental laser cycle:

$$
\begin{aligned}
\mathrm{e}^{\mathrm{i} n \omega_{0} t}-\mathrm{e}^{\mathrm{i} n\left(\omega_{0} t+\pi\right)} & =\mathrm{e}^{\mathrm{i} n \omega_{0} t}\left(1-\mathrm{e}^{\mathrm{i} n \pi}\right) \\
& = \begin{cases}2 \mathrm{e}^{\mathrm{i} n \omega_{0} t} & \text { if } n \text { is odd }, \\
0 & \text { if } n \text { is even. }\end{cases}
\end{aligned}
$$

This is, in a nutshell, atomic HHG. The classical concepts introduced in this section are sufficient to extend the recollision model to solids, a task that is performed in the next section.

\subsection{From atoms to solids}

To introduce a model of HHG in solids, it is convenient to translate the atomic model, presented as electrons moving in real space, in momentum/energy space. This is the natural framework in which solids are described. How to understand the interaction of the atom with the strong laser field in this framework?

2.2.1. Atomic $H H G$ in momentum space. The energy diagram of atoms in strong laser fields is shown to the left of figure 3. In the following, only one electron-hole pair is considered, which emits high harmonics at the energy determined by its time of creation. The electron is initially in the ground state of the atom with energy $-I_{p}$. Upon tunnelling (blue arrow labelled ' 1 '), the electron is freed in the vacuum with approximately zero initial velocity. Because the energy of a free electron is $T=k^{2} / 2$, it occupies the lowestenergy state in this continuum function of momentum (a parabolic band, black solid line in the middle of figure 3). As the electron accelerates, it gains the momentum described by equation (1), which can be rewritten in terms of the vector potential of the laser field $A(t)(F(t)=-\partial A / \partial t)$

$$
k(t)=A(t)-A\left(t^{\prime}\right) .
$$

In doing so, the electron gains the energy $k^{2}(t) / 2$ : it climbs up the parabolic band (blue arrow labelled ' 2 '). The hole, created in the ground state upon ionization, also gains the same momentum as the electron ${ }^{4}$, but its energy is constant. At the time of recollision $t_{r}$, the electron recombines with the hole (undergoing a vertical transition back to the ground state, marked by the blue arrow labelled ' 3 ') at the momentum $k\left(t_{r}\right)=A\left(t_{r}\right)-A\left(t^{\prime}\right)$ and a photon of energy equal to $k^{2}\left(t_{r}\right) / 2+I_{p}$ is emitted.

2.2.2. The model for solids. In crystalline solids, contrary to atoms, the electron is initially in a continuum of states, the valence band. The valence band is separated from higher energetic states by an energy gap, or minimum band gap (equivalent to the atom's ionization potential). In the following, only dielectric or semiconducting materials will be considered, so that the minimum band gap is non zero. The excited states, the conduction bands, also form several continua. The formation of bands reflects the quantum-

4 Electrons and holes move together in momentum space. To describe the hole, in fact, the prescription of solid state physics says that both the charge and the mass of the electron must change sign. Since $k \propto e / m A(t)$, the momentum is the same for electrons and holes. 
mechanical electron's de-localization over the lattice of the crystal. The motion of electrons is therefore different than in free space, and results in the energy bands being nonparabolic functions of momentum. To the right of figure 3 , one valence and one conduction band of a $\mathrm{ZnO}$ crystal are plotted.

The recollision model of $\mathrm{HHG}$ from solids is the following.

1. Around the peak of the laser field, an electron tunnels vertically from the valence to the conduction band (red arrow labelled ' 1 ' in figure 3 ) at the momentum of highest tunnelling probability. This is illustratively at $k=0$ in figure 3 to make contact with the atomic case, but it can be at $k \neq 0$. An electron-hole pair is thus formed. The rate of tunnelling is obviously different than in atoms, and is currently a matter of intense debate in the scientific community $[10,11,23,24]$.

2. Regardless on how the electron tunnels, the laser field subsequently accelerates the pair. Like in atoms, its momentum is determined by equation (5). However, in a solid, $k$ is the crystal momentum; it is not to be confused with the mechanical momentum. The crystal momentum does not directly describe the motion of the pair in real space. The energy also increases (red arrow labelled '2'), according to the structure of the band. The evolution of the momentum is plotted in figure 4(a) for some electron-hole pairs created at different times after the peak of the laser field. The same plot would be obtained for the case of an isolated atom. Like in atoms, the electron and the hole accelerate in opposite directions in real space (see figure 4(b)).

3. Some time later in the laser cycle, the electron can reencounter the hole with non-zero momentum, they can recombine (red arrow labelled ' 3 ' in figure 3 ) and emit a harmonic photon of energy $\hbar \omega=\varepsilon_{g}\left[k\left(t_{r}\right)\right]$, with $\varepsilon_{g}(k)$ being the difference in energy between the conduction and valence states at the momentum $k$. The time of reencounter and the associated momentum of each electron-hole pair is marked by open circles in figure 4(a).

The time of re-encounter is dictated by the motion of electrons and holes in real space, which is markedly different between atoms and solids. This is because, in the solid state language, the velocity of electrons in band $m$ (proportional to their mechanical momentum) is determined by (in atomic units):

$$
v_{m}(k)=\nabla_{k} E_{m}(k),
$$

where $E_{m}(k)$ is the momentum-dependent energy of the band in which the electron (or hole) travels. For an electron in vacuum, for which $E(k)=k^{2} / 2$, the mechanical momentum equals the crystal momentum: $v=k$. Therefore, no meaningful distinction is made between velocity and momentum. However, because bands in solids are more complex functions of momentum, this simple relation does not hold. To describe the motion of electrons and holes in real space, one has to solve equation (1) (or equation (5)) together with: $x_{e}(t)=\int_{t^{\prime}}^{t} \nabla E_{c}[k(\tau)] \mathrm{d} \tau ; \quad x_{h}(t)=\int_{t^{\prime}}^{t} \nabla E_{v}[k(\tau)] \mathrm{d} \tau$,

where $E_{c}$ and $E_{v}$ are the conduction and valence bands respectively. Figure 4(b) shows the real space trajectories of electrons (solid lines) and holes (dashed lines) for two different times of creation of the pairs (red for pairs created at the peak of the field, and black after the peak, corresponding to emission at the cut-off energy). They are compared to the atomic trajectory responsible for high harmonic emission at the cut-off (green lines). The motion in the solid is clearly different than in vacuum. For example: the time of reencounter, determined by the crossing of the dashed with the solid lines, is markedly different between solids and atoms (compare the black and green lines). Similar deviations are found for all other trajectories (except for $t^{\prime}=0$ ). Also, the trajectories have a strong anharmonic component. In fact, the current arising from the acceleration of electrons and holes in their respective bands is another source of harmonics, competing with the recollision mechanism. This source will be discussed in section 3 .

As a result of the 'generalized' time of recollision, and of the band structure, the energy of recolliding pairs maps differently than in atoms to their time of creation. This is shown in figure 4(c). Contrary to atoms, where the kinetic energy of the electron is potentially unlimited, in a solid composed of only two bands, the maximum harmonic emission is capped to the maximum bandgap energy (marked by the black dashed line).

2.2.3. Consequences of the model. In summary, the recollision model for solids generalizes atomic HHG to arbitrary band structures (not only parabolic). In atoms, the electrons' trajectories are universally defined, once all quantities are scaled with the laser peak field strength and frequency. In solids, the universality is lost: the trajectories also depend on the band structure, and to which portion the electron-hole pairs explore during their motion. The generalized relation between the times of creation, of recollision, and the harmonic photon energy, is the most apparent consequence of the model. It will be investigated further in a next section. Other paradigms of atomic HHG are lost as well. Below, we briefly discuss some of them.

- The hole also moves. This is a consequence of the dispersion of the band in momentum space. In atoms, the ground state is an almost flat band. Therefore, according to equation (6) the hole's velocity is almost zero.

- The hole's and electron's maximum excursion is limited. Because typical bands change curvature at sufficiently high momenta, the velocity reaches a maximum at the inflection point. Therefore, an increase in momentum slows down the particle propagating in the band, and limits its maximum excursion in the laser field. Figure 5 shows the maximum excursion (in units of lattice cells) 

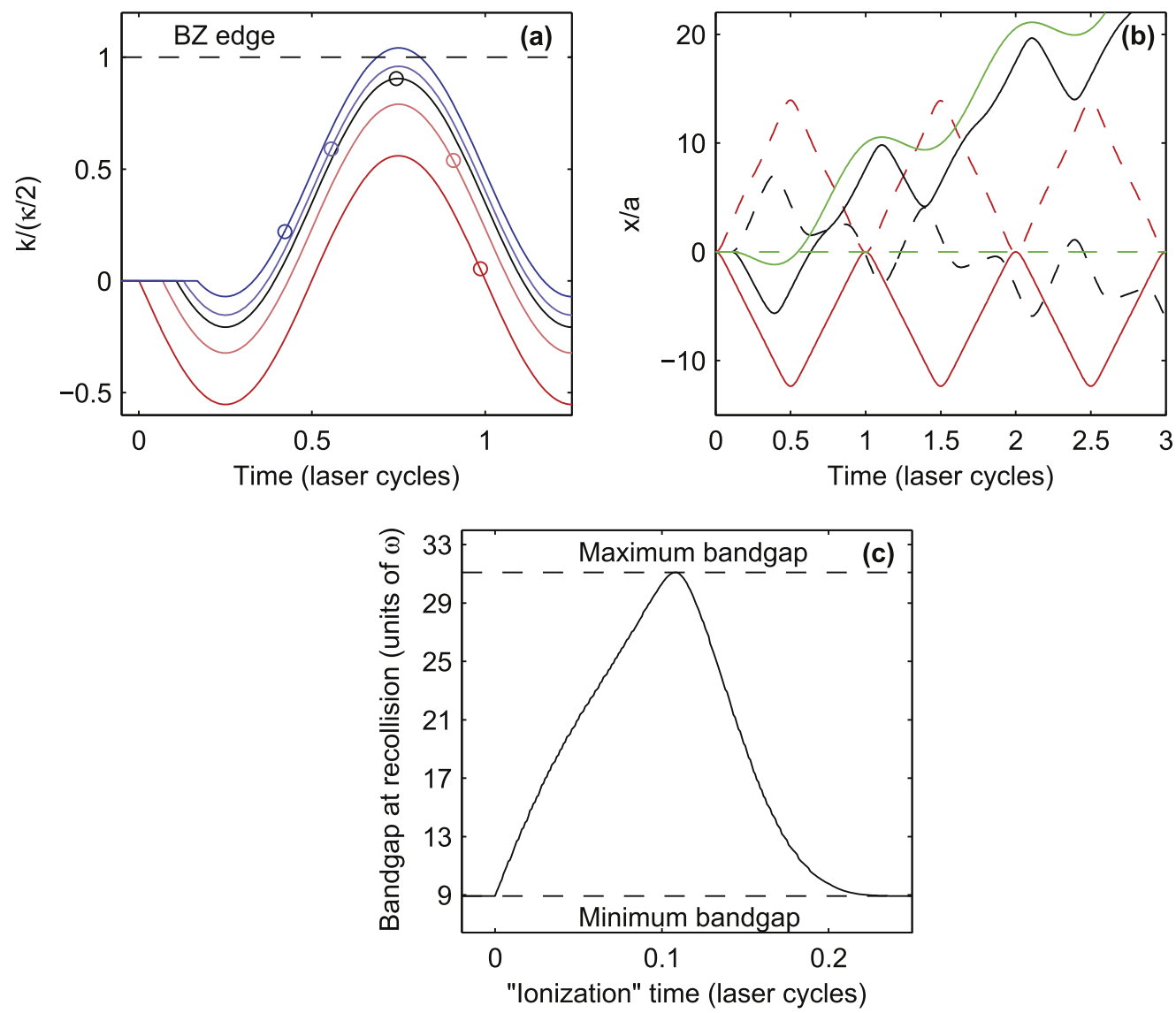

Figure 4. Semiclassical propagation in solids. (a) Time-dependent momentum $k(t)$ (calculated with equation (5) for a set of trajectories born at different times after the peak of the field. The dark red line is for $t^{\prime}=0$, while the black line marks the trajectory that emits the highest harmonic photon energy (cutoff). The time of recollision between electrons and hole is marked for each trajectory by the open circles. Momentum is given in units of half the reciprocal lattice vector $\kappa / 2=\pi / a_{l}$, with $a_{l}$ the separation between the lattice planes identified by the particular relative orientation of the laser polarization with respect to the crystallographic axis. Here, $a_{l}=5.32$ a.u. for $\mathrm{ZnO}$. The dashed line marks the edge of the first Brillouin Zone at $k=\kappa / 2$. In a periodic solid, owing to the discrete translational symmetry, higher momenta can be mapped back to the first BZ. The simulation is performed with laser frequency $\omega_{0}=0.014$ a.u. $(\lambda=3.25 \mu \mathrm{m})$, and $F_{0}=0.0046$ a.u. $\left(0.24 \mathrm{~V} \AA^{-1}\right.$, corresponding to an intensity of $\left.8 \times 10^{11} \mathrm{~W} \mathrm{~cm}^{-2}\right)$. (b) Position of electrons (solid lines) and holes (dashed lines) following their creation, for pairs born at the peak of the field (red lines) and for the cutoff trajectory (black lines). The green lines are the cutoff atomic trajectories, for an $800 \mathrm{~nm}$ laser at $10^{14} \mathrm{~W} \mathrm{~cm}^{-2}$ (as in figure 2). (c) Energy of electron-hole pairs at the time of recollision, as a function of the time of their creation. This energy is transferred to high harmonic photons, upon recombination of the electrons with the holes. Classically allowed emission is limited within the minimum and maximum bandgap of the material (dashed lines), typically occurring at the centre and edge of the BZ respectively. (C) 2015 IEEE. Adapted, with permission, from [25].

for electrons and holes born at the peak of the field as a function of the peak field strength (in atomic units).

- Recollision can happen away from the origin. Figure 6 shows the position of electron-hole pairs at recollision (in units of lattice cells) with respect to their place of origin (at $x=0$ ) for all trajectories born around the peak of the laser field. Pairs can recollide some lattice cells away!

- Trajectories born before the peak of the laser electric field can recollide. When the field strength is increased, the trajectories significantly distort: some pairs that would typically recollide, will not, and pairs born before the peak of the field, which would typically avoid recollision, recollide. An in-depth analysis of the distortions is deferred to section 3 .

In the next section, the semiclassical model of HHG in solids is derived from a quantum-mechanical theory.

\section{Quantum model}

The model is, again, a generalization of the one used to describe atomic HHG. Just like in the atomic case, high harmonics are emitted by the oscillating dipole. There are two major results: (i) the semiclassical recollision model already introduced can be derived from one part of the oscillating dipole, and (ii) there are other parts that also emit high harmonics. The relative importance and the temporal properties of harmonics emitted by each mechanism are analysed in the last part of the section.

\subsection{Derivation of the dipole moment}

The time dependent Hamiltonian is given by $H(t)=$ $H_{0}-\mathbf{x} \cdot \mathbf{F}(t)$, where $H_{0}=T+U$ is the unperturbed Hamiltonian with $T=-\mathbf{p}^{2} / 2$ the kinetic energy operator, 


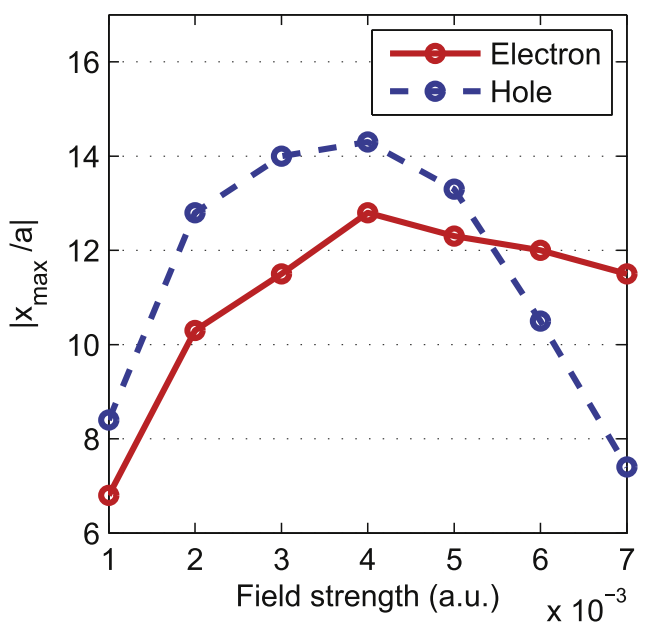

Figure 5. Maximum excursion of electrons (red line) and holes (blue line) in units of lattice cells, as a function of field strength. (c) 2015 IEEE. Adapted, with permission, from [25].

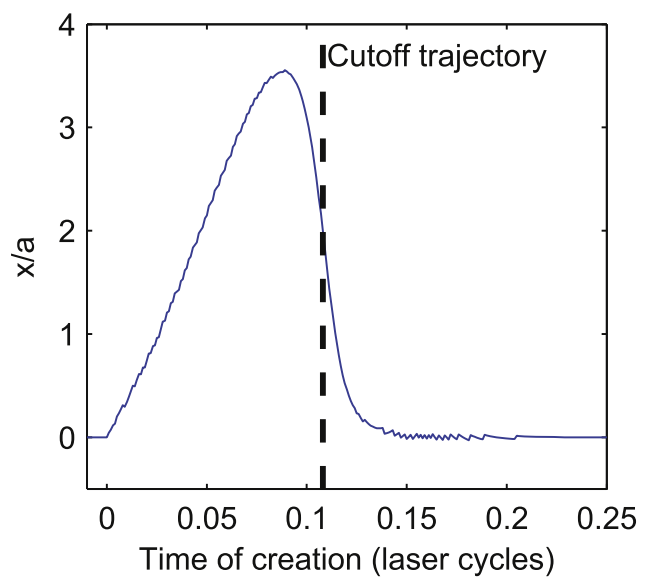

Figure 6. Position of recollision between electrons and holes of each trajectory born between the peak and the first zero of the field. The dashed vertical line marks the cut-off trajectory. (C) 2015 IEEE. Adapted, with permission, from [25].

$\hat{\mathbf{p}}=-\mathrm{i} \boldsymbol{\nabla}$ the momentum operator, and $U(\mathbf{x})$ the periodic potential of the lattice.

The field free Hamiltonian $H_{0}$ has Bloch eigenstates $\Phi_{m, \mathbf{k}}(\mathbf{x})=u_{m, \mathbf{k}}(\mathbf{x}) \exp (\mathbf{i k} \cdot \mathbf{x})$ with energies $E_{m, \mathbf{k}}=E_{m}(\mathbf{k})$ in band $\mathrm{m}$ with crystal momentum $\mathbf{k} ; u_{m, \mathbf{k}}$ is the periodic part of the Bloch function.

In the presence of the laser field the wavefunction becomes time dependent. In the length gauge it is represented as

$$
\Psi(\mathbf{x}, t)=\sum_{m} \int_{\mathrm{BZ}} a_{m}(\mathbf{k}, t) \Phi_{m, \mathbf{k}}(\mathbf{x}) \mathrm{d}^{3} \mathbf{k}
$$

with probability amplitudes $a_{m}(\mathbf{k}, t)$. The integration is performed over the primitive cell of the momentum space, the Brillouin Zone (BZ). The wavefunctions are normalized such that:

$$
\int_{\mathbb{R}^{3}}\left|\Phi_{m, \mathbf{k}}(\mathbf{x})\right|^{2} \mathrm{~d} \mathbf{x}=V
$$

where $V$ is the volume of the crystal. To obtain the equations of motion for the probability amplitudes $a_{m}(\mathbf{k}, t)$, ansatz (8) is substituted into the time-dependent Schrödinger equation for the Hamiltonian $H(t)$ :

$$
\mathrm{i} \frac{\partial \Psi(\mathbf{x}, t)}{\partial t}=H(t) \Psi(\mathbf{x}, t)
$$

and the Bloch eigenstates are integrated out, yielding

$$
a_{m}=\left(-\mathrm{i} E_{m}(\mathbf{k})+\mathbf{F}(t) \nabla_{\mathbf{k}}\right) a_{m}+\mathrm{i} \mathbf{F}(t) \sum_{m^{\prime} \neq m} \mathbf{d}_{m m^{\prime}}(\mathbf{k}) a_{m^{\prime}}
$$

with

$$
\mathbf{d}_{m m^{\prime}}(\mathbf{k})=\mathrm{i} \int \mathrm{d}^{3} \mathbf{x} u^{*}{ }_{m, \mathbf{k}}(\mathbf{x}) \nabla_{\mathbf{k}} u_{m^{\prime}, \mathbf{k}}(\mathbf{x})
$$

the transition dipole moment between bands $m$ and $m^{\prime}$. In the derivation, the relation $\left\langle\Phi_{m, \mathbf{k}}|\mathbf{x}| \Phi_{m^{\prime}, \mathbf{k}^{\prime}}\right\rangle=-\mathrm{i} \partial_{\mathbf{k}} \delta_{m m^{\prime}}+\mathbf{d}_{m m^{\prime}}(\mathbf{k})$ [26] is used, which splits the transition dipole into an intraband and interband contribution, respectively ${ }^{5}$.

According to equation (11) there are two ways the electron population is transferred to band $m$ and momentum k:

1. from the other bands within the states of same momentum. The term proportional to $\mathbf{d}_{m m^{\prime}}$ is responsible for this transition. The same term causes transitions in two-level systems.

2. from adjacent momentum states within the same band. The term proportional to $\nabla_{\mathbf{k}}$ causes this transition. It describes the acceleration of electrons by the laser field.

High harmonics are emitted by the oscillating dipole (or rather its time derivative, the current):

$$
\begin{aligned}
\langle\Psi|\mathbf{x}| \Psi\rangle= & \sum_{\mathbf{k} \in \mathrm{BZ}}\left[-\mathrm{i} \sum_{m} \nabla_{\mathbf{k}}\left|a_{m}(\mathbf{k}, t)\right|^{2}\right. \\
& \left.+\sum_{m \neq m^{\prime}} a_{m^{\prime}}^{*}(\mathbf{k}, t) a_{m}(\mathbf{k}, t) \mathbf{d}_{m m^{\prime}}(\mathbf{k})\right] .
\end{aligned}
$$

The first term on the RHS deals with electrons in each separate band. It is commonly called intraband, although it necessarily takes into account all bands, at least during the tunnelling step. The second term arises from the interference between electrons in bands $m$ and $m^{\prime}$. It is referred to as interband.

3.1.1. Relation to atomic HHG. The model introduced is a generalization of that used for atomic HHG [27]. In fact, a set of differential equations equivalent to equation (11) can be obtained by considering only two bands: one with the dispersion of electrons in vacuum $E_{c}=\mathbf{k}^{2} / 2$ and the other with ground state energy $E_{v}=-I_{p}$. Accordingly, $\Phi_{m, \mathbf{k}}$ for the ground state is replaced with the ground state wavefunction $\Phi_{g}$ (and drop the summation over $\mathbf{k}$ ). In atoms, the intraband dipole contributes only to low order harmonics, whereas

\footnotetext{
5 The fact that the intraband term in $\left\langle\Phi_{m, \mathbf{k}}|\mathbf{x}| \Phi_{m^{\prime}, \mathbf{k}^{\prime}}\right\rangle$ return the operator $\nabla_{\mathbf{k}}$ rather than a number is somewhat bothering. The correct understanding of this expression can be found in [26].
} 
higher order harmonics-arising from the recolliding electron-hole pairs - are generated by the interband term.

3.1.2. The density matrix formulation. Rather than working with the Schrödinger equation, one could work with the density matrix formalism [28, 29]. With this approach, dephasing of the interband polarization, that may have a strong effect on the high harmonic spectrum (as shall be seen later), can be included more easily. For a generic statistical mixture of states $|\Psi\rangle=\sum_{j} p_{j}\left|\Psi_{j}\right\rangle$, the density matrix is defined as:

$$
\rho \doteq \sum_{j} p_{j}\left|\Psi_{j}\right\rangle\left\langle\Psi_{j}\right|
$$

For the pure state of equation (8), the elements of the density matrix can be related to the probability amplitudes:

$$
\begin{aligned}
\rho & =\sum_{\mathbf{k}, \mathbf{k}^{\prime} \in \mathrm{BZ}} \sum_{m, m^{\prime}} a_{m}(\mathbf{k}, t) a_{m^{\prime}}^{*}\left(\mathbf{k}^{\prime}, t\right)\left|\Phi_{m, \mathbf{k}}\right\rangle\left\langle\Phi_{m^{\prime}, \mathbf{k}^{\prime}}\right| \\
& =\sum_{\mathbf{k}, \mathbf{k}^{\prime} \in \mathrm{BZ}} \sum_{m, m^{\prime}} \rho_{m m^{\prime}}\left(\mathbf{k}, \mathbf{k}^{\prime}, t\right)\left|\Phi_{m, \mathbf{k}}\right\rangle\left\langle\Phi_{m^{\prime}, \mathbf{k}^{\prime}}\right| .
\end{aligned}
$$

In this formalism, the intraband $\mathbf{j}_{\mathrm{ra}}$ and interband $\mathbf{j}_{\mathrm{er}}$ contribution to the current are [30]:

$$
\begin{aligned}
\mathbf{j}_{\mathrm{ra}}(t)= & \sum_{m} \int_{\overline{\mathrm{BZ}}} \mathbf{v}_{m}[\mathbf{K}+\mathbf{A}(t)] \rho_{m m}(\mathbf{K}, t) \mathrm{d}^{3} \mathbf{K}, \\
\mathbf{j}_{\mathrm{er}}(t)= & \frac{\mathrm{d}}{\mathrm{d} t} \sum_{m \neq m^{\prime}} \int_{\overline{\mathrm{BZ}}} \mathbf{d}_{m m^{\prime}} \\
& \times[\mathbf{K}+\mathbf{A}(t)] \rho_{m m^{\prime}}(\mathbf{K}, t) \mathrm{d}^{3} \mathbf{K}+\text { c.c. }
\end{aligned}
$$

where $\mathbf{v}_{m}(\mathbf{k})=\nabla_{\mathbf{k}} E_{m}(\mathbf{k})$ is the velocity of electrons in band $m$, $\rho_{m m}=n_{m}$ is the population of band $m$ and $\rho_{m m^{\prime}}$ determines the interband polarization between bands $m$ and $m^{\prime}$. The crystal momentum $\mathbf{k}$ has been transformed into a frame moving with the vector potential $-\mathrm{d} \mathbf{A} / \mathrm{d} t=\mathbf{F}, \mathbf{K}=\mathbf{k}-\mathbf{A}(t)$. As a result, the first Brillouin zone is also shifted to $\overline{\mathrm{BZ}}=\mathrm{BZ}-\mathbf{A}(t)$. The high-harmonic spectrum is obtained from the Fourier transform (FT) of $\mathbf{j}_{\mathrm{t}}=\mathbf{j}_{\mathrm{ra}}+\mathbf{j}_{\mathrm{er}}$, as $\left|\mathrm{FT}\left\{\mathbf{j}_{\mathrm{t}}\right\}\right|^{2}$.

A set of differential equations for the populations and the polarization can be derived from equation (11) by introducing the following definitions:

$$
\begin{aligned}
b_{m}(\mathbf{K}, t) & =a_{m}(\mathbf{K}, t) \mathrm{e}^{\mathrm{i} \int_{-\infty}^{t} E_{m}\left[\mathbf{K}+\mathbf{A}\left(t^{\prime}\right)\right] \mathrm{d} t^{\prime}}, \\
n_{m} & =\left|b_{m}\right|^{2}, \quad \pi_{m m^{\prime}}=b_{m} b_{m^{\prime}}^{*} .
\end{aligned}
$$

to obtain [31]:

$$
\begin{aligned}
\dot{\pi}_{m m^{\prime}}= & -\frac{\pi_{m m^{\prime}}}{T_{2}}+\mathrm{i} \Omega_{m m^{\prime}}^{*}\left(n_{m}-n_{m^{\prime}}\right) \mathrm{e}^{-\mathrm{i} S_{m m^{\prime}}} \\
& +\mathrm{i} \sum_{m^{\prime \prime} \neq\left\{m, m^{\prime}\right\}}\left(\Omega_{m^{\prime} m^{\prime \prime}} \pi_{m m^{\prime \prime}} \mathrm{e}^{\mathrm{i} S_{m^{\prime} m^{\prime \prime}}}-\Omega_{m m^{\prime \prime}}^{*} \pi_{m^{\prime} m^{\prime \prime}}^{*} \mathrm{e}^{\left.-\mathrm{i} S_{m m^{\prime \prime}}\right)},\right. \\
\dot{n}_{m}= & \mathrm{i} \sum_{\mathrm{m}^{\prime \prime} \neq\left\{m, m^{\prime}\right\}} \Omega_{m m^{\prime}} \pi_{m m^{\prime}} \mathrm{e}^{\mathrm{i} S_{m m^{\prime}}}+\text { c.c., }
\end{aligned}
$$

where $\Omega_{m m^{\prime}}(\mathbf{K}, t)=\mathbf{F}(t) \cdot \mathbf{d}_{m m^{\prime}}[\mathbf{K}+\mathbf{A}(t)]$ is the Rabi frequency. $S_{m m^{\prime}}$ is the classical action:

$$
S_{m m^{\prime}}(\mathbf{K}, t)=\int_{-\infty}^{t} \varepsilon_{m m^{\prime}}\left[\mathbf{K}+\mathbf{A}\left(t^{\prime}\right)\right] \mathrm{d} t^{\prime}
$$

where $\varepsilon_{m m^{\prime}}=E_{m}-E_{m^{\prime}}$ is the bandgap between bands $m$ and $m^{\prime}$. The dephasing $\left(T_{2}\right)$ term has been added phenomenologically; in principle, it could also be derived more formally by extending the theory to account for coupling to phonon bath and impurities and for electron-electron scattering [28]. Equations (18) are subject to the constraint $\sum_{m} n_{m}=1$. Equations (18) are general for any number of bands in a solids. For the remainder of the tutorial, unless where explicitly stated, the analysis is restricted to two-bands with valence $(m=v)$ and conduction $(m=c)$ bands, but simulations with more bands are possible [16, 31, 32].

3.1.3. Relation to the optical bloch equations. With the definitions $n_{m}=\left|a_{m}\right|^{2}$ and $\Pi=a_{c} a_{v}^{*}$, equation (11) become the Semiconductor Bloch equations [29]:

$$
\begin{aligned}
\dot{\Pi}(\mathbf{k}, t)= & F(t) \nabla_{\mathbf{k}} \Pi(\mathbf{k}, t)-\mathrm{i} \varepsilon_{g}(\mathbf{k}) \Pi(\mathbf{k}, t) \\
& -\mathrm{i} \Omega(\mathbf{k}, t) w(\mathbf{k}, t), \\
\dot{n}_{m}(\mathbf{k}, t)= & F(t) \nabla_{\mathbf{k}} n_{m}(\mathbf{k}, t)-2 \operatorname{Im}\left\{\Omega(\mathbf{k}, t) \Pi^{*}(\mathbf{k}, t)\right\} .
\end{aligned}
$$

They differ from the Optical Bloch equations that describe two level systems by the terms $\propto \nabla_{\mathbf{k}}$, which are responsible for the acceleration of the electrons and holes in their respective bands. In other words, a two-bands periodic solid can be understood as a collection of coupled two-level systems, each system labelled by a different momentum, where the laser field transfers population between the systems.

We note that the term 'Semiconductor Bloch equations' is used in the literature in different contexts. Originally it referred to the equations without the $\nabla_{\mathbf{k}}$ term but with exchange interactions between electrons [28]. Lately, it has become more common to refer to the above equations also as Semiconductor Bloch equations.

3.1.4. The analytical solution. In order to better understand the physical processes driving intraband and interband HHG in solids, it is useful to explore equation (18) by using the Keldysh approximation [33], $w(t) \approx 1$ in equation $(18 b)$. This decouples equation (18) so that they can be formally integrated. Inserting the result into equation (16), the spectra of the currents are obtained:

$$
\begin{aligned}
& \mathbf{j}_{\mathrm{ra}}(\omega)= \sum_{m=c, v} s_{m} \int_{-\infty}^{\infty} \mathrm{d} t \mathrm{e}^{-\mathrm{i} \omega t}\left[\int_{\mathrm{BZ}} \mathrm{d}^{3} \mathbf{k} \mathbf{v}_{m}(\mathbf{k})\right. \\
& \times \int_{-\infty}^{t} \mathrm{~d} t^{\prime} F\left(t^{\prime}\right) \mathrm{d}^{*}\left(\boldsymbol{\kappa}_{t^{\prime}}\right) \\
& \times \int_{-\infty}^{t^{\prime}} \mathrm{d} t^{\prime \prime} F\left(t^{\prime \prime}\right) \mathrm{d}\left(\boldsymbol{\kappa}_{t^{\prime \prime}}\right) \\
&\left.\times \mathrm{e}^{\mathrm{i} S\left(\mathbf{k}, t^{\prime \prime}, t^{\prime}\right)-\left(t^{\prime}-t^{\prime \prime}\right) / T_{2}}+\text { c.c. }\right], \\
& \mathbf{j}_{\mathrm{er}}(\omega)=\omega \int_{-\infty}^{\infty} \mathrm{d} t \mathrm{e}^{-\mathrm{i} \omega t}\left[\int_{\mathrm{BZ}} \mathrm{d}^{3} \mathbf{k} \mathbf{d}^{*}(\mathbf{k}) \int_{-\infty}^{t} \mathrm{~d} t^{\prime} F\left(t^{\prime}\right)\right. \\
& \times \\
&\left.\mathrm{d}\left(\boldsymbol{\kappa}_{t^{\prime}}\right) \mathrm{e}^{\mathrm{i} S\left(\mathbf{k}, t^{\prime}, t\right)-\left(t-t^{\prime}\right) / T_{2}}+\text { c.c. }\right],
\end{aligned}
$$

where $\boldsymbol{\kappa}_{t^{\prime}}=\mathbf{k}+\mathbf{A}\left(t^{\prime}\right)-\mathbf{A}(t)$. Further, the crystal momentum 
has been transformed back to the initial frame $\mathbf{k}=\mathbf{K}+\mathbf{A}(t)$, and $\quad S\left(\mathbf{k}, t^{\prime}, t\right)=\int_{t^{\prime}}^{t} \varepsilon_{\mathrm{g}}\left(\boldsymbol{\kappa}_{\tau}\right) \mathrm{d} \tau$. Note that deriving equation (20) directly from equation (11) would yield the same result except for the dephasing term. For the range of fields $F_{0} \leqslant 0.008$ a.u., explored in experiments [14], the harmonic spectra obtained from equation (20) and by using the full twoband equation (18) are practically identical. The Keldysh approximation might even be more robust in solids than in atoms because, contrary to atoms, it is difficult to achieve more than few percent in ionization without causing damage.

\subsection{Interband harmonics and recollisions}

The connection between the semiclassical model of recollisions in solids and the interband current is demonstrated here.

3.2.1. Theoretical derivation. equation (20b) includes integrals of two oscillating functions: $F\left(t^{\prime}\right)$ and the complex exponential, with phase:

$$
\mathrm{i} \phi=\mathrm{i} S\left(\mathbf{k}, t^{\prime}, t\right)-\mathrm{i} \omega t-\left(t-t^{\prime}\right) / T_{2} .
$$

$\phi$ oscillates faster than the electric field, because in typical experiments $\omega_{0} \ll E_{g} \quad\left(E_{g}\right.$ is the minimum bandgap). Therefore, the integral would not build up, unless $\phi$ remains constant for some time. One can approximate the integrals with the saddle point method, which calculates the integrand around the points where the phase is stationary. These points are determined by:

$$
\begin{gathered}
\frac{\mathrm{d} \phi}{\mathrm{d} t^{\prime}}=\varepsilon_{\mathrm{g}}\left[\mathbf{k}-\mathbf{A}(t)+\mathbf{A}\left(t^{\prime}\right)\right]-\frac{\mathrm{i}}{T_{2}}=0, \\
\nabla_{\mathbf{k}} \phi=\int_{t^{\prime}}^{t} \Delta \mathbf{v}(\mathbf{k}-\mathbf{A}(t)+\mathbf{A}(\tau)) \mathrm{d} \tau=0, \\
\frac{\mathrm{d} \phi}{\mathrm{d} t}=\varepsilon_{\mathrm{g}}(\mathbf{k})-\omega+\frac{\mathrm{i}}{T_{2}}=0,
\end{gathered}
$$

where $\Delta \mathbf{v}(\mathbf{k})=\nabla_{\mathbf{k}} \varepsilon_{\mathrm{g}}(\mathbf{k})=\mathbf{v}_{c}-\mathbf{v}_{v}$ is the difference of the velocities of the electron and the hole in their respective bands. To simplify the discussion it is useful to consider the limit $T_{2}=\infty$. As shall be seen later, neglect of dephasing does not alter the physical picture down to $T_{2} \approx 1 \mathrm{fs}$. The above equations yield the following intuitive semiclassical description of interband harmonics.

1. Because $\varepsilon_{g}(\mathbf{k}) \geqslant E_{g} \geqslant 0$, equation ( $\left.22 a\right)$ can only be solved for complex $t^{\prime}$. The imaginary part of $t^{\prime}$ describes the tunnelling of electrons from the valence to the conduction band. Solution of this equation has been obtained only for simple [24] and approximate [33] band structures. However, one can neglect the influence of the tunnelling step by setting $E_{g}=0$. In this case, by writing $\varepsilon_{g}(\mathbf{k})=E_{g}+h(\mathbf{k})$, with $h \geqslant 0$ and $h\left(\mathbf{k}_{0}\right)=0$, equation (22a) reads $h\left[\mathbf{k}-\mathbf{A}(t)+\mathbf{A}\left(t^{\prime}\right)\right]=0$. The latter is solved for:

$$
\mathbf{k}=\mathbf{k}_{0}+\mathbf{A}(t)-\mathbf{A}\left(t^{\prime}\right),
$$

where $\mathbf{k}_{0}$ is the momentum at the minimum direct band gap. The equation reduces to equation (5) in the case that the minimum direct band gap is at zero crystal momentum $\left(\mathbf{k}_{0}=0\right)$. The solution of equation (22a), in summary, implies that electron-hole pairs, after their birth through tunnelling across a direct band gap at $\mathbf{k}=\mathbf{k}_{0}$, accelerate in the reciprocal space according to equation (23).

2. $\Delta \mathbf{x}_{m}=\int_{t^{\prime}}^{t} \mathbf{v}_{m} \mathrm{~d} \tau=\mathbf{x}_{m}(t)-\mathbf{x}_{m}\left(t^{\prime}\right)$ is the distance propagated by electrons/holes in the conduction/valence band ( $m=c, v)$ between time of birth $t^{\prime}$ and time of observation $t$. Therefore, equation $(22 b)$, reads $\nabla_{\mathbf{k}} \phi=$ $\Delta x_{c}-\Delta x_{v}=0$. Since electrons and holes are born at the same position, the condition implies that high harmonics are emitted only upon re-encounter of each electron with its associated hole. Truthfully, electrons and holes are not quite exactly born at the same position, but at the exit of the tunnel. This quantummechanical aspect can be grasped by keeping the imaginary components of the stationary points, as clearly illustrated in [34] for the atomic case.

3. Finally, equation $(22 c)$ is conservation of energy. Upon recollision at time $t$, each electron recombines with its associated hole and emits a high harmonic photon with energy equal to the bandgap at the time of recollision: $\omega=\varepsilon_{g}\left[\mathbf{A}(t)-\mathbf{A}\left(t^{\prime}\right)\right]$.

To conclude the derivation, it should be stressed that the quantum-mechanical aspects of HHG can only be retained by solving equation (22) in the complex plane, by adapting the procedure outlined in [34] to solids. For example, in atoms, because the electron exits the tunnel offset from the hole at position $x_{0}$, it needs to travel a little more to recollide with it. During this extra bit of time it acquires the extra energy $F_{0} x_{0}$, therefore extending the quantum-mechanical cut-off beyond the classical limit of $3.17 U_{p}$. This and other effects will also play a role in solids.

3.2.2. Results of the simulations. The semiclassical model of the interband polarization is validated by numerical integration of equation (18). The time-dependent inter- $\left(\mathbf{j}_{\mathrm{er}}\right)$ and intraband $\left(\mathbf{j}_{\mathrm{ra}}\right)$ currents are then calculated with equation (16). Their spectrum is reported in figure 7 for two different values of the dephasing time $T_{2}$. Details about the simulation are reported in the appendix. The spectrum of both currents exhibit an extended plateau from the minimum bandgap of the material (corresponding to the 9th harmonic order) up to the 25 th order (both orders are marked by blacked dashed vertical lines). The plateau in the interband current arises from the recollision and recombination of electron-hole pairs. Later it will be shown that the plateau in the intraband current also bears the signature of recollisions.

To demonstrate the role of recollisions in the plateau of the interband current, one can look at the variation of the harmonic frequency with time in this spectral region, the hallmark of the recollision process as introduced in section 1. For the purpose, a windowed Fourier transform is performed 


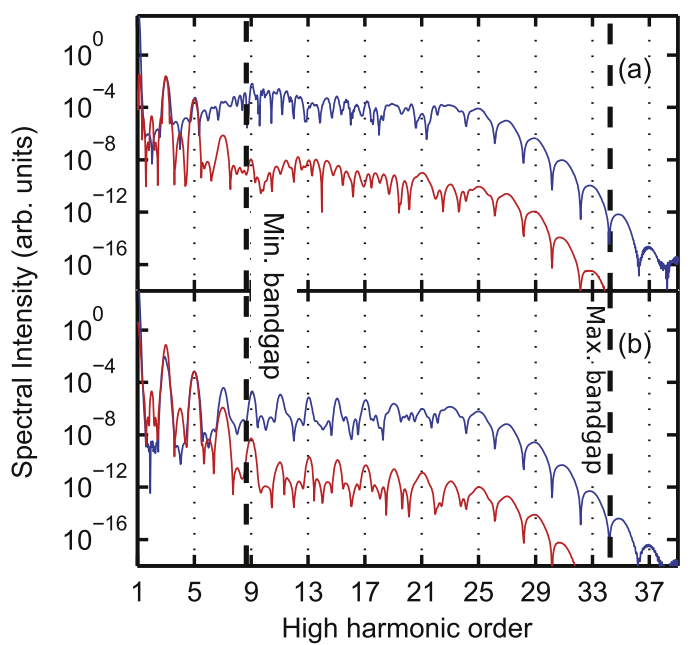

Figure 7. Harmonic spectrum for interband $\left(\left|F T\left\{\mathbf{j}_{\text {er }}\right\}\right|^{2}\right.$, blue line $)$ and intraband $\left(\left|F T\left\{\mathbf{j}_{\mathrm{ra}}\right\}\right|^{2}\right.$, red line) currents for a field strength $F_{0}=0.003$ a.u.; the cosine laser field with $\omega_{0}=0.014$ a.u., corresponding to a laser period $T_{0}=2 \pi / \omega_{0}=10.9 \mathrm{fs}$, is multiplied by a temporal Gaussian envelope with a FWHM of ten cycles. The dephasing time is (a) $T_{2}=\infty$ and (b) $T_{2}=T_{0} / 4$. The laser polarization is along the $\Gamma-M$ direction of the reciprocal space of a wurtzite $\mathrm{ZnO}$ crystal. Details about the crystal and its band structure are given in the appendix. The dashed vertical black lines mark the minimum band gap at the centre of the Brillouin zone (at $3.4 \mathrm{eV}$ ) and the maximum band gap at the edge of the Brillouin Zone (at $13 \mathrm{eV}$ ). Adapted with permission from [35]. Copyright 2015 by the American Physical Society.

in the following way:

$$
\mathbf{j}(\omega, \tau)=\int_{-\infty}^{\infty} W(t-\tau) \mathbf{j}(t) \mathrm{e}^{-\mathrm{i} \omega t} \mathrm{~d} t .
$$

Here, the interband time-dependent dipole is multiplied by a Blackman window function $W$ which is narrow compared to the fundamental laser cycle (the window is only 0.34 cycles wide). The spectrum of the resulting dipole is recorded as a function of the delay between the window and the laser cycle, thereby returning a $2 \mathrm{D}$ map of the emission in time and frequency-a spectrogram.

The spectrograms are shown in figures 8(e)-(h) for increasing field strengths (from (e) to (h)). The spectral intensity of the interband dipole is colour coded. At each laser half-cycle, the high harmonic frequency increases, reaches a maximum, and decreases. The solid white line, instead, is the prediction of the semiclassical model. Panel (f) shows the same data as in figure 4(c), plotted as a function of time rather than birth time. The near perfect agreement between the simulated spectrum and the prediction of the semiclassical model confirms that recolliding electron-hole pairs are responsible for the generation of interband high harmonics, at the given laser frequency.

There is a slight discrepancy however: the simulated cutoff is slightly higher than in the semiclassical calculation. This deviation possibly arises from neglecting the initial offset of the electron from the hole as a result of tunnelling.

A thorough validation of the semiclassical model can be performed with the aid of simulations. Panels (a)-(d) show the semi-classically calculated harmonic order as a function of time for the same field strengths of the right panels. Electrons born at a specific time can recollide with the hole multiple times. These higher returns are colour coded from the first (blue) to the fifth (black). The thin green line in panels (a), (b) represents the energy of the first recollision in an atomic system, for a field strength that yields the same cut-off; for the atomic system it is assumed that the ionization potential $I_{p}=E_{g}$. In panels (e), (h) only recollisions within the first laser cycle are plotted (solid white line). Several points can be made.

- Existence of generalized trajectories. Whereas the atomic trajectories are independent of the field strength (as illustrated in section 1), in a crystal they are not. For example the blue line in figure $8(\mathrm{~b})$, calculated with $F_{0}=0.0046$ a.u., is distorted with respect to that of figure $8(\mathrm{a})$, where $F_{0}=0.003$ a.u. For the higher field, the cut-off originates from trajectories that are born and recollide almost $10^{\circ}$ later in the cycle as compared to the lower field case. The differences between solids and atoms become even more pronounced as the field is further increased, see figures 8(c), (d). This striking difference is a consequence of the non-parabolic band dispersion. For higher field strengths, electrons and holes start to explore the non-quadratic part of the band. When this happens, the quadratic energy-momentum relation and therewith the velocity is strongly modified and the trajectories are no longer invariant with respect to the field strength.

Similar to atomic HHG, the cut-off energy separates two sets of trajectories that recollide within one optical cycle and contribute to the same harmonic order. Those that travel a longer time, the long trajectories, are born after the peak of the field but before the birth time of the cut-off trajectory $t_{c}^{\prime}$; the short trajectories instead are born at times later than $t_{c}^{\prime}$.

- The cut-off can saturate. For field strengths $F_{0} \leqslant 0.0046$ a.u., the cut-off is below or equals the maximum possible photon energy (black dashed-dotted line), which is determined by the maximum energy difference between valence and conduction bands. The long and short branches are clearly identifiable. When the intensity is increased further, see figures 8(c), (d), the cut-off saturates. As a result, the simple long/short trajectory picture is lost. For $F_{0}=0.0058$ a.u., figure $8(\mathrm{c})$, the same electron-hole pairs undergo up to three recollisions near the cutoff energy before the long trajectories recollide the first time.

For $F_{0}=0.008$ a.u., figure $8(\mathrm{~d})$, long trajectories do not recollide at all, but short trajectories can recollide a second and a third time within the optical cycle. This field strength corresponds to $\sim 0.43 \mathrm{~V}^{-1}$ in the crystal. With the refractive index $n=1.8992$ of $\mathrm{ZnO}$ at $\lambda=3.62 \mu \mathrm{m}$, it corresponds to $F_{\text {vac }}=0.62 \mathrm{~V}^{-1}$, which has been reached in experiments [14]. 


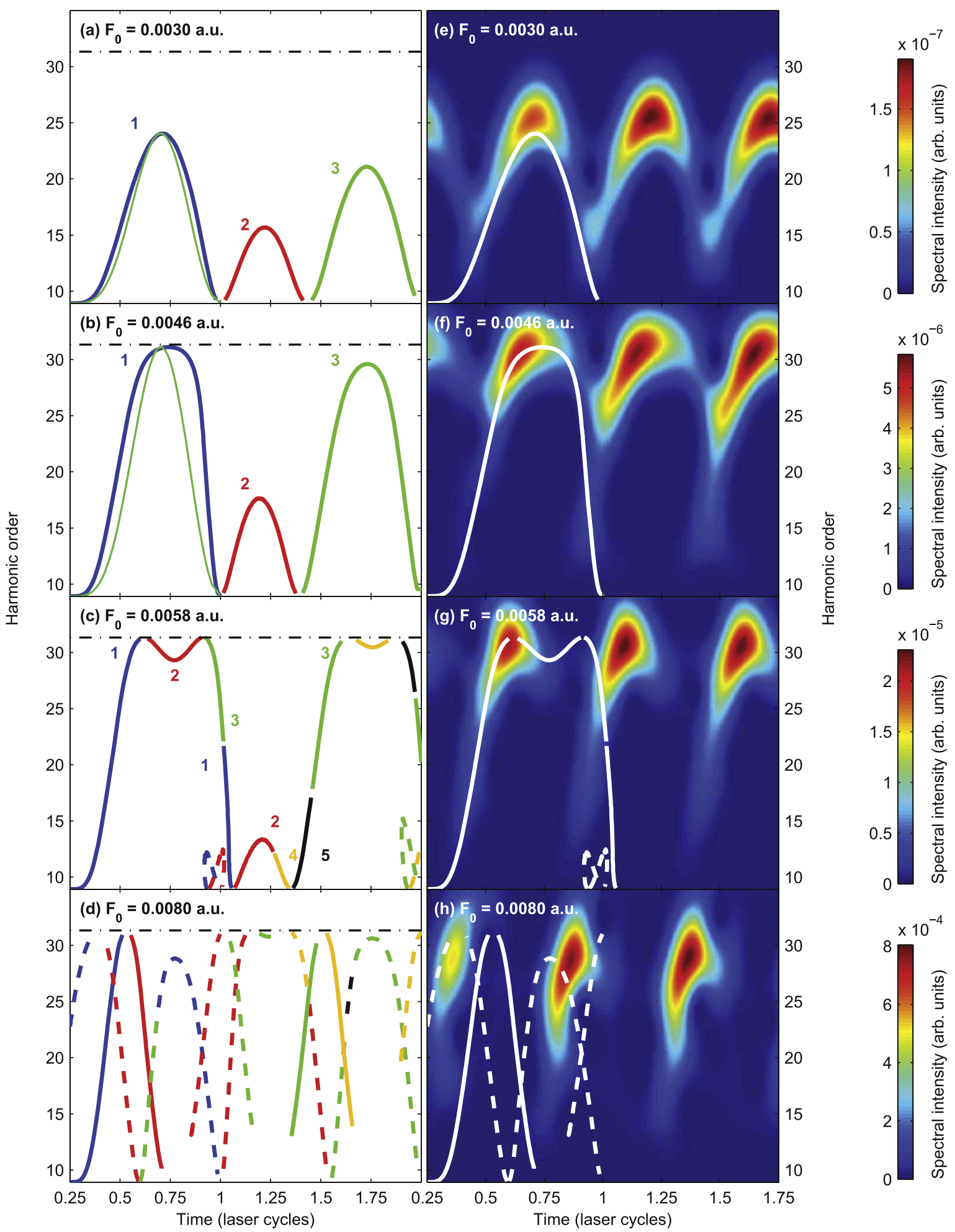

Figure 8. ((a)-(d), left panels) Semiclassical calculation of the high harmonic photon energy as a function of time for generation in a ZnO crystal at various field strengths. Subsequent recollision events are numbered sequentially and colour coded as follows: first (blue), second (red), third (green), fourth (yellow), fifth (black). Solid (dashed) lines are for pairs created after (before) the peak of the field. The thin green line in panels (a), (b) is the recollision energy of atomic high harmonics normalized to the cut-off of the crystal case. The dashed-dotted black line is the maximum bandgap at the edge of the Brillouin Zone. ((e)-(h), right panels) Simulated high harmonic spectral intensity of interband emission (colour code) as a function of harmonic order (vertical axis) and time (horizontal axis). The classical trajectories recolliding within the cycle, found in (a)-(d), are the solid and dashed white lines. Replicas of this emission at following laser half-cycles arise from the intrinsic periodicity of the generation process. 


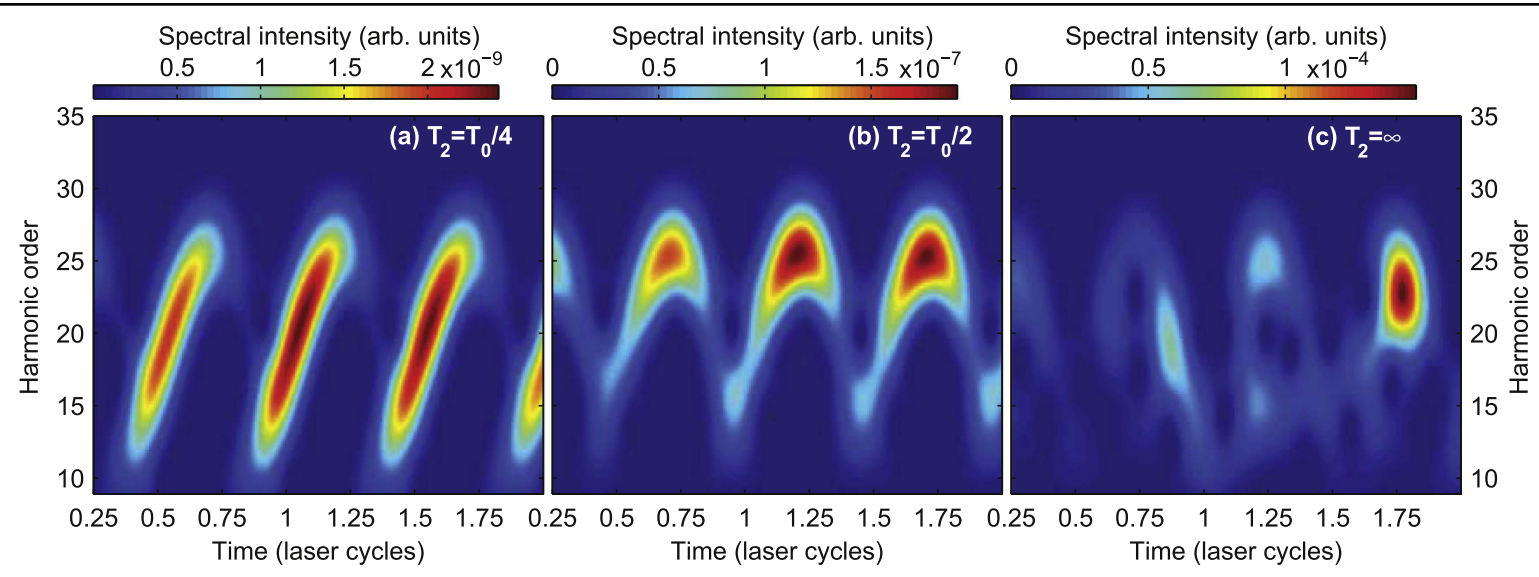

Figure 9. Harmonic order versus time for three different dephasing times: (a) $T_{2}=T_{0} / 4$, (b) $T_{2}=T_{0} / 2$, (c) $T_{2}=\infty$. The laser frequency is $\omega_{0}=0.014$ a.u. and the field strength is $F_{0}=0.003$ a.u. The shorter the dephasing time the weaker the long trajectories are. In (c) multiple returns significantly modify the recollision.

- Trajectories born before the peak can recollide. For $F_{0}=0.008$ a.u., harmonic emission is shifted at earlier times starting from the field zero (at 0.25 cycles, marked by the dashed blue line in panel (d)). This emission is associated to recolliding trajectories born before the peak of the field. In the atomic case, this can only happen if the Coulomb attraction between electrons and holes is considered [36]. Excitonic interaction is not included in the simulations presented here, so this effect arises solely from the generalized motion of electron-hole pairs in solids.

3.2.3. Role of dephasing. Another discrepancy is the absence in the simulations of multiple recollisions, labelled '2' and ' 3 ' in figures 8(a), (b). In atomic HHG, long returns are (i) suppressed by quantum diffusion of the wavepacket [27], which results in a small recombination cross section between free electrons and the atomic ground state and (ii) difficult to detect because their large dipole phase results in extremely curved phase fronts, ultimately leading to highly divergent beams [37].

In solids, in addition to the atomic effects, short dephasing times could contribute to the suppression of higher-order returns. Dephasing also weakens the long trajectory contribution. This is apparent from the trajectory analysis of figure 9 , for $T_{2}=T_{0} / 4$ (a), $T_{0} / 2$ (b) and $T_{2}=\infty$ (c). For increasing dephasing times the spectral intensity of the long trajectories becomes stronger. When the dephasing time is long enough to allow returns longer than a laser cycle (c), multiple recollisions interfere and, together with the varying intensity of the pulse envelope, lead to aperiodic photon emission ${ }^{6}$. This results in the loss of the clear harmonic structure in the spectrum, as shown in figure 7(a).

The simulation suggests that dephasing could play an important role in HHG. Intrinsic dephasing times in

6 If a CW laser is used instead, the emission is still periodic and only the odd harmonics constructively interfere. However, multiple returns modulate their intensity. semiconductors are found to be in the 15-50 fs range by photon echo experiments [38, 39]; however, these experiments only probe low energy electrons near zero momentum. Under the strong laser field, electrons can be excited to very high energies. An estimate of dephasing times at higher electron energies can be obtained from electron diffraction experiments. The measured scattering length for a $6 \mathrm{eV}$ electron is generally on the order of $100 \AA$ [40], corresponding to a scattering time of about $7 \mathrm{fs}$. Furthemore, recent transient absorption experiments performed in silicon [11] are consistent with electron-electron scattering times $<1$ fs when the sample is excited by a strong near-infrared pulse.

\subsection{Mechanisms of intraband emission}

There are several mechanisms responsible for intraband emission. The intraband current is defined by equation $(16 a)$ :

$$
\mathbf{j}_{\mathrm{ra}}(t)=\sum_{m=c, \mathrm{v}} \int_{\overline{\mathrm{BZ}}} \mathbf{v}_{m}[\mathbf{K}+\mathbf{A}(t)] n_{m}(\mathbf{K}, t) \mathrm{d}^{3} \mathbf{K} .
$$

First, harmonics are emitted by the nonlinearity of the band velocities $\mathbf{v}_{m}[\mathbf{K}+\mathbf{A}(t)]$, which results in the nonlinear oscillations of electrons and holes represented by the black line in figure 10(a). When the electron is accelerated across the entire Brillouin Zone, it undergoes a Bloch oscillation. Consequently, the emitted harmonics are dubbed Bloch harmonics. However, the electron need not to be accelerated so strongly to generate harmonics. Therefore, to avoid confusion, these harmonics will be named velocity harmonics. They have been extensively studied in the literature in the context of HHG [14-16] and even unequivocally identified in experiments conducted with $\mathrm{THz}$ pump wavelengths [41]. Their spectrum can be analytically solved [29], and the motion characterized by the dimensionless parameter $\omega_{B} / \omega$, where $\omega_{B}=F d$ (in a.u.) is the Bloch frequency. The spectrum of $\mathbf{v}_{m}[\mathbf{A}(t)]$ (therefore for electrons that tunnel at $\mathbf{K}=0$ ) from a $\mathrm{ZnO}$ crystal is reported in figure 10(b) (black line). The windowed Fourier transform (figure 10(c)) reveals that these harmonics are emitted in phase as discrete bursts at each peak of the laser field, when the acceleration of the electron is 

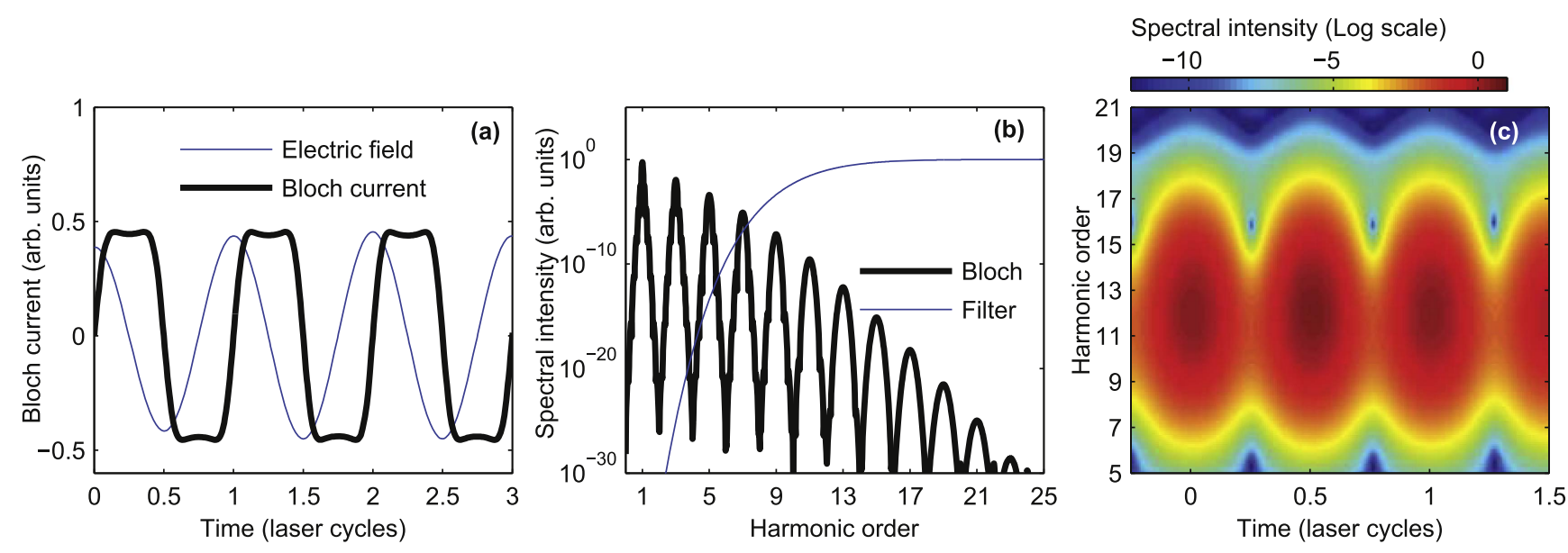

Figure 10. Properties of velocity harmonics. (a) Electrons (black line) move asynchronously with the laser field (blue line) because their velocity is determined by the band dispersion. (b) The spectrum of $\mathbf{v}_{m}[\mathbf{A}(t)]$ (black line) decreases monotonically with harmonic order. (c) The spectrogram (spectral intensity is in logarithmic scale) reveals emission in phase at each field crest. The blue line in panel (b) filters out the dominant low-order harmonics in the windowed Fourier transform. The laser frequency is $\omega_{0}=0.014$ a.u. and the field strength is $F_{0}=0.003$ a.u.
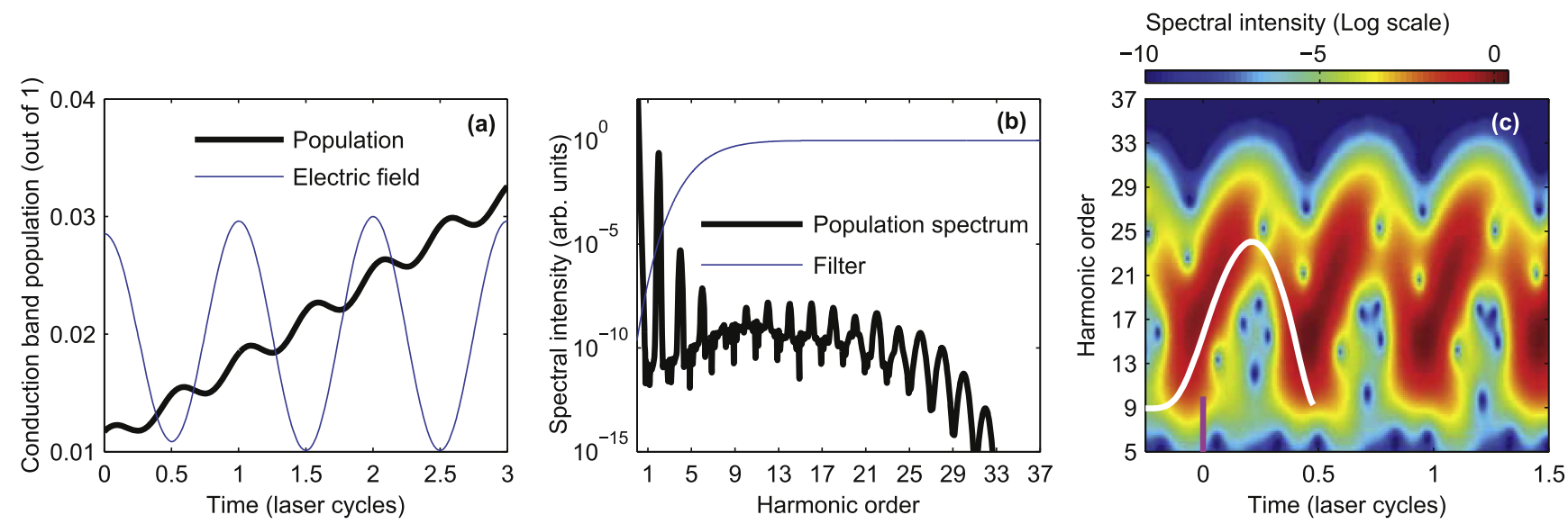

Figure 11. The conduction band population (black line in panel (a)) increases almost step-like at each field crest (laser field is the blue line). The dynamics generate a broad harmonic plateau (panel (b), black line), and low order harmonics of rapidly decreasing intensity. Harmonics in the plateau are emitted at the time of recollision (solid white line in panel (c)), whereas low order harmonics are all emitted in phase (dashed magenta line). The laser frequency is $\omega_{0}=0.014$ a.u. and the field strength is $F_{0}=0.003$ a.u.

maximum (see panel (a)). The absence of spectral phase results from the time-reversal symmetry of the oscillations. The properties of the Fourier transform, in fact, demand that if $\mathbf{v}_{m}(t)=\mathbf{v}_{m}(-t)$, then $\mathbf{v}_{m}(\omega)=\mathbf{v}^{*}{ }_{m}(\omega)$, where $*$ indicates complex conjugation. Therefore, $\mathbf{v}_{m}(\omega) \in \mathbb{R}$.

Second, harmonics arise from the nonlinearity of tunnel ionization, embedded in $n_{m}(\mathbf{K}, t)$, that results in almost steplike increase (decrease) in the conduction (valence) band population. The momentum-integrated population in the conduction band, $n_{c}(t)$, is plotted in figure 11(a). It is obtained by setting $\mathbf{v}_{m}[\mathbf{K}+\mathbf{A}(t)]=1$ in equation (25). The generation of harmonics by means of tunnel ionization was predicted by Brunel for the atomic case [42]. Typically, the spectrum consists of low-order harmonics of rapidly decreasing intensity (see panel (b), black line, up to the 9th harmonic). Panel (c) shows that Brunel harmonics are emitted in phase (marked by the dashed magenta line, up to the 9th harmonic order).
Third, the intraband current also contains a recollisionlike contribution that depends on the polarization buildup between electrons and holes. This contribution arises from the exponential term containing the semiclassical action $S$ in equation (20a). Like Brunel emission, it is also embedded in $n_{m}(\mathbf{K}, t)$. However, it gives rise to a broad spectral plateau in figure 11(b), all in all similar to the interband one. In fact, these harmonics are emitted at the time of recollision, as shown by the solid white line in panel (c). The position of the crossover between Brunel harmonics and the recollision-like mechanism, at $\omega \sim E_{g}$ in figure 11, depends on the dephasing time. For short dephasing times, recollision is less significant because of the loss of coherence between electrons and holes. Therefore, the plateau is weaker and the crossover shifts to higher harmonics.

In conclusion, only recolliding electron-hole pairs give rise to a variation of the emitted high harmonic order in time. 


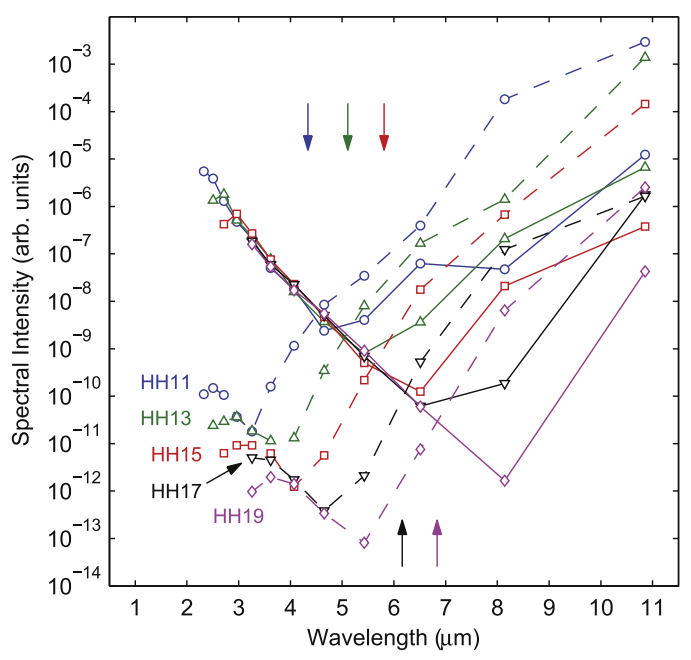

Figure 12. Theoretical prediction of harmonic intensity versus laser wavelength for various high harmonic orders; circles, triangles pointed upward, squares, triangles pointed downward, and diamonds refer to order $11,13,15,17$, and 19, respectively. The solid and dashed lines show the interband and intraband contributions for $T_{2}=2.7 \mathrm{fs}\left(T_{0} / 4\right.$ for $\left.\lambda=3.25 \mu \mathrm{m}\right)$, respectively. The field strength is kept constant at $F_{0}=0.003$ a.u. The laser polarization is along the $\Gamma-M$ direction of a wurtzite $\mathrm{ZnO}$ crystal. Details about the crystal and the band structure are given in the appendix. The arrows mark the wavelength above which a given harmonic falls below the minimum bandgap. Adapted with permission from [35]. Copyright 2015 by the American Physical Society.

For all other mechanisms, harmonic emission is in phase. This feature will be exploited to find the signature of recolliding hole-pairs in the experiment presented in the next chapter.

\subsection{Relative importance of interband and intraband currents}

Figure 7 predicts that interband harmonics above the minimum bandgap of the material are orders of magnitude stronger than intraband harmonics. Figure 12 investigates how interband (solid lines) and intraband (dashed lines) contributions scale with laser wavelength, for a fixed field strength $F_{0}=0.003$ a.u. The arrows mark the wavelengths above which a given harmonic order falls below the minimum band gap. For the chosen dephasing time $\left(T_{2}=T_{0} / 4\right)$, as long as high harmonics are above the band gap, interband emission dominates. For shorter dephasing times, intraband harmonics gain more weight and the points where interband and intraband emission intersect move slowly to shorter wavelengths; however, down to $T_{2} \approx 1 \mathrm{fs}$, interband emission remains dominant over most of the displayed wavelength range. The crossing point also depends on field strength. It varies by about $1 \mu \mathrm{m}$ with field strengths in the range $F_{0}=0.003-0.1$ a.u.

The interband contribution drops exponentially with increasing wavelength. This is potentially a signature of dephasing: in equation $(20 b)$, as the excursion time $t-t^{\prime}$ increases with $\lambda$, the amplitude decreases $\propto \exp \left[\left(t-t^{\prime}\right) / T_{2}\right]$. The longer the electron is separated from the hole, the higher the chance to endure collisions, which lessen the coherence they have with respect to one another.

The wavelength dependence of the intraband yield is more complex. It most likely results from a mixing of the different mechanisms identified above and can even increase for longer wavelengths. For wavelengths below $\sim 5 \mu \mathrm{m}$, intraband emission drops as fast as interband emission. Here, harmonics come from the recollision-like mechanism, and are therefore as prone to dephasing. At longer wavelengths, velocity or Brunel harmonics dominate, as indicated by the rapid decrease of spectral intensity with harmonic order. Here, the intraband contribution increases with wavelength. This increase is possibly caused by electrons and holes traversing the Brillouin Zone more than once in a laser halfcycle, and therefore undergoing more oscillations in the same cycle fraction. Therefore, the spectral intensity increases.

In summary, the analysis indicates a clear distinction between two regimes: for wavelengths below $\sim 5 \mu \mathrm{m}$, interband harmonics dominate, whereas intraband emission from Bloch or Brunel mechanisms dominates for longer excitation wavelengths. The prediction seems in line with recent experiments performed at $10 \mu \mathrm{m}$ wavelength, where the signature of Bloch oscillations has been found in the simultaneous emission of harmonics at the peaks of the driving laser field $[16,41]$.

\subsection{Role of diffusion}

In atomic $\mathrm{HHG}$, after its birth through ionization, the electron diffuses in space such that at the time of recollision its width can be several times larger than the hole, which remains highly localized on the ion. Recollisions become increasingly disfavoured with increasing diffusion, reflecting the lower chance that the electron hits the localized hole. In solids, however, the hole also diffuses and one might argue that the increased overlap with the electron should mitigate the decrease in HHG efficiency. In the following, we argue that this is not the case and explain why.

The role of diffusion is easiest understood in the coordinates orthogonal to the laser polarization, which are unaffected by the laser field. The saddle point equation $(22 b)$ in the momenta orthogonal to the laser polarization reads:

$$
\nabla_{\mathbf{k}_{\{x, y\}}} \phi=\int_{t^{\prime}}^{t} \Delta \mathbf{v}_{\{x, y\}}(\mathbf{k}-\mathbf{A}(t)+\mathbf{A}(\tau)) \mathrm{d} \tau=0 .
$$

Note that the saddle point condition implies that the electron and the hole need to occupy the same site at time $t$ : $x_{c}(t)-x_{c}\left(t^{\prime}\right)=x_{v}(t)-x_{v}\left(t^{\prime}\right)\left(\right.$ with $\left.x_{v}\left(t^{\prime}\right)=x_{c}\left(t^{\prime}\right)\right)$.

To simplify the discussion, let's assume that the electronhole wavepacket always occupies the parabolic part of the bandgap in $\mathbf{k}_{\{x, y\}}$. Therefore, $\Delta \mathbf{v}_{\{x, y\}}(\mathbf{k}-\mathbf{A}(t)+\mathbf{A}(\tau))=$ $\Delta \mathbf{v}_{\{x, y\}}(\mathbf{k})=\mathbf{k}_{\{x, y\}} / m_{\{x, y\}}$, where $m_{\{x, y\}}^{-1}=\nabla_{\mathbf{k}_{\{x, y\}}}^{2} \varepsilon_{g}(\mathbf{k})$. The saddle point is then:

$$
\int_{t^{\prime}}^{t} \frac{\mathbf{k}_{\{x, y\}}}{m_{\{x, y\}}} \mathrm{d} \tau=\frac{\mathbf{k}_{\{x, y\}}}{m_{\{x, y\}}}\left(t-t^{\prime}\right)=0 \quad \leftrightarrow \quad \mathbf{k}_{\{x, y\}}^{\text {st }}=0 .
$$


Saddle point integration of the interband current (equation (20b)) over $\mathbf{k}_{x}$ and $\mathbf{k}_{y}$ yields:

$$
\begin{aligned}
\mathbf{j}_{\mathrm{er}}(\omega)= & \omega \int_{-\infty}^{\infty} \mathrm{d} t \mathrm{e}^{-\mathrm{i} \omega t}\left[\int_{-\infty}^{t} \mathrm{~d} t^{\prime} \frac{2 \pi \sqrt{m_{x} m_{y}}}{t-t^{\prime}}\right. \\
& \left.\times \int_{\mathrm{BZ}} \mathrm{d} \mathbf{k}_{\mathbf{z}} G\left(\mathbf{k}, t, t^{\prime}\right)+\text { c.c. }\right], \\
G\left(\mathbf{k}, t, t^{\prime}\right) & =\mathbf{d}^{*}(\mathbf{k}) F\left(t^{\prime}\right) d\left(\boldsymbol{\kappa}_{t^{\prime}}\right) \mathrm{e}^{\mathrm{i} S\left(\mathbf{k}, t^{\prime}, t\right)-\left(t-t^{\prime}\right) / T_{2}} .
\end{aligned}
$$

The prefactor $\sqrt{m_{x} m_{y}} /\left(t-t^{\prime}\right)$ results from diffusion of the wavepackets, which is expressed by the accumulated phase. The longer the excursion time $t-t^{\prime}$, the lower the current. Even if the electron and the hole diffuse at the same rate, that is if $m_{c}=-m_{v}$, where $\{c, v\}$ refer to the conduction and valence bands respectively, the reduced effective mass is nonzero:

$$
\frac{1}{m_{x}}=\frac{1}{m_{c, x}}-\frac{1}{m_{v, x}}=\frac{1}{m_{e}}+\frac{1}{m_{h}}=\frac{2}{m_{e}} .
$$

Therefore, although the electron's and hole's wavepackets always overlap, HHG still suffers from diffusion. This is because the electron-hole pair acquires a phase as it travels along $x$ or $y$ that oscillates fast in momentum space. As a result, all possible paths with non-zero transverse momentum cancel out in the integration - they are phase-mismatched. Physically, if electrons and holes are born with the same non-zero crystal momentum, they have an opposite transverse non-zero velocity which separates them from each other at a constant rate. A linearly polarized laser field will never be able to collide them. Exact integration is trivial and yields the same result.

With this analysis, HHG efficiency is higher for localized electrons and holes than for diffused wavepackets, even if electron and hole wavefunctions overlap perfectly over the whole space.

\section{Experimental evidence of recollisions}

This section is devoted to show that high harmonics can be generated by recolliding electron-hole pairs. Not only this finding opens the possibility to extend to solids the attosecond methods developed to study molecules, but may allow to tackle questions specific to the solid phase, such as dephasing, ionization and damage.

The signature of recollisions is obtained by perturbing the HHG process with a second harmonic field, thereby producing even harmonics. We show that second harmonic fields as weak as few $\mathrm{V} \mu \mathrm{m}^{-1}$, below the DC breakdown threshold of typical semiconductors, are sufficient to produce even harmonics. This extreme sensitivity to perturbing fields opens the possibility to integrate electronics with high harmonics. In our view, this is another relevant distinction with the atomic case.

\section{1. $H H G$ from $Z n O$}

4.1.1. The experimental setup. The experimental setup is sketched in figure 13. The laser source delivers mid-infrared laser pulses with centre wavelength of $3.76 \mu \mathrm{m}$, energy of $60 \mu \mathrm{J}$ and pulse duration of $95 \mathrm{fs}$. These mid-infrared pulses are obtained by difference frequency generation in a $400 \mu \mathrm{m}$ thick $\mathrm{AgGaS}_{2}$ (AGS) crystal between the signal and idler of a commercial Optical Parametric Amplifier (LightConversion HE-Topas-C) pumped by $4.5 \mathrm{~mJ}$ of Ti:Sapphire $800 \mathrm{~nm}$ laser pulses. The output of the OPA is further amplified in a beta barium borate crystal pumped by $10 \mathrm{~mJ}$ of $800 \mathrm{~nm}$ pulses. The mid-infrared beam is spatially filtered with a pin-hole.

High harmonics are generated by focusing the midinfrared pulses with $f / 30$ spherical $\mathrm{Ag}$ mirror into a $500 \mathrm{~nm}$ thick $\mathrm{ZnO}$ single crystal, wurtzite phase, epitaxially grown over a $0.5 \mathrm{~mm}$ thick sapphire substrate. Both crystals are oriented with their $c$-axis, or the [0001] direction, aligned along the laser $k$-vector. The sample is oriented with the sapphire side facing the incoming beam. The pulse energy incident on the sample is $19 \mu \mathrm{J}$. The peak intensity inside the sample is $0.85 \mathrm{TW} \mathrm{cm}^{-2}$ $\left(0.25 \mathrm{~V}^{-1}\right)$, which is calculated from the vacuum intensity accounting for reflection loss: $F_{0}=2 F_{\mathrm{vac}} /(n+1)$. Here, $n=1.8971$ is the refractive index of $\mathrm{ZnO}$ at the mid-infrared wavelength. The vacuum intensity is calculated by independently measuring the pulse energy, duration, and the focus size. The pulse duration is measured with a dispersion-free SHG frequency-resolved optical gating after the $\mathrm{ZnO}$ crystal. The focus size is measured with the knife-edge technique. The fundamental spectrum before and after the crystal is not visibly different, demonstrating that self-phase modulation is negligible. Likewise, because the incident peak power $P=1.76 \times 10^{8} \mathrm{~W}$ is below the critical power for selffocusing $P_{\mathrm{cr}}=2.2 \times 10^{8} \mathrm{~W}$ (assuming a nonlinear refractive index for Sapphire of $n_{2}=5.4 \times 10^{-21} \mathrm{~W}$ [1]), self-focusing should also be negligible.

The high harmonics are focused with a spherical Al mirror on the entrance slit of a visible-ultraviolet spectrometer from OceanOptics. The detected wavelength range is from 200 to $900 \mathrm{~nm}$. Figure 14 shows the harmonic signal with respect to the fundamental intensity. An initial sharp rise in harmonic signal is followed by a much slower increase, in agreement with a previous experiment [14]. The scaling is roughly irrespective of the harmonic order, in contrast with predictions of conventional nonlinear optics. Thus, the harmonics are non-perturbative.

To determine if high harmonics originate from a recollision mechanism, a weak second harmonic field is added to the strong mid-infrared fundamental field. The second harmonic is generated in a $300 \mu \mathrm{m}$ thick AGS crystal optimized for Type-I generation, positioned right after the pinhole to exploit the high intensity of the focussed fundamental beam and its high quality resulting from the spatial filter. The two colours are separated and recombined with dichroic beam splitters that reflect the second harmonic. The polarization of the latter is rotated parallel to the fundamental before the recombining mirror. The energy of the second harmonic is controlled with an iris. The delay between the two colours is varied with a PZT stage on the second harmonic arm.

The addition of the second harmonic breaks the symmetry between successive half-cycles of the laser field. 


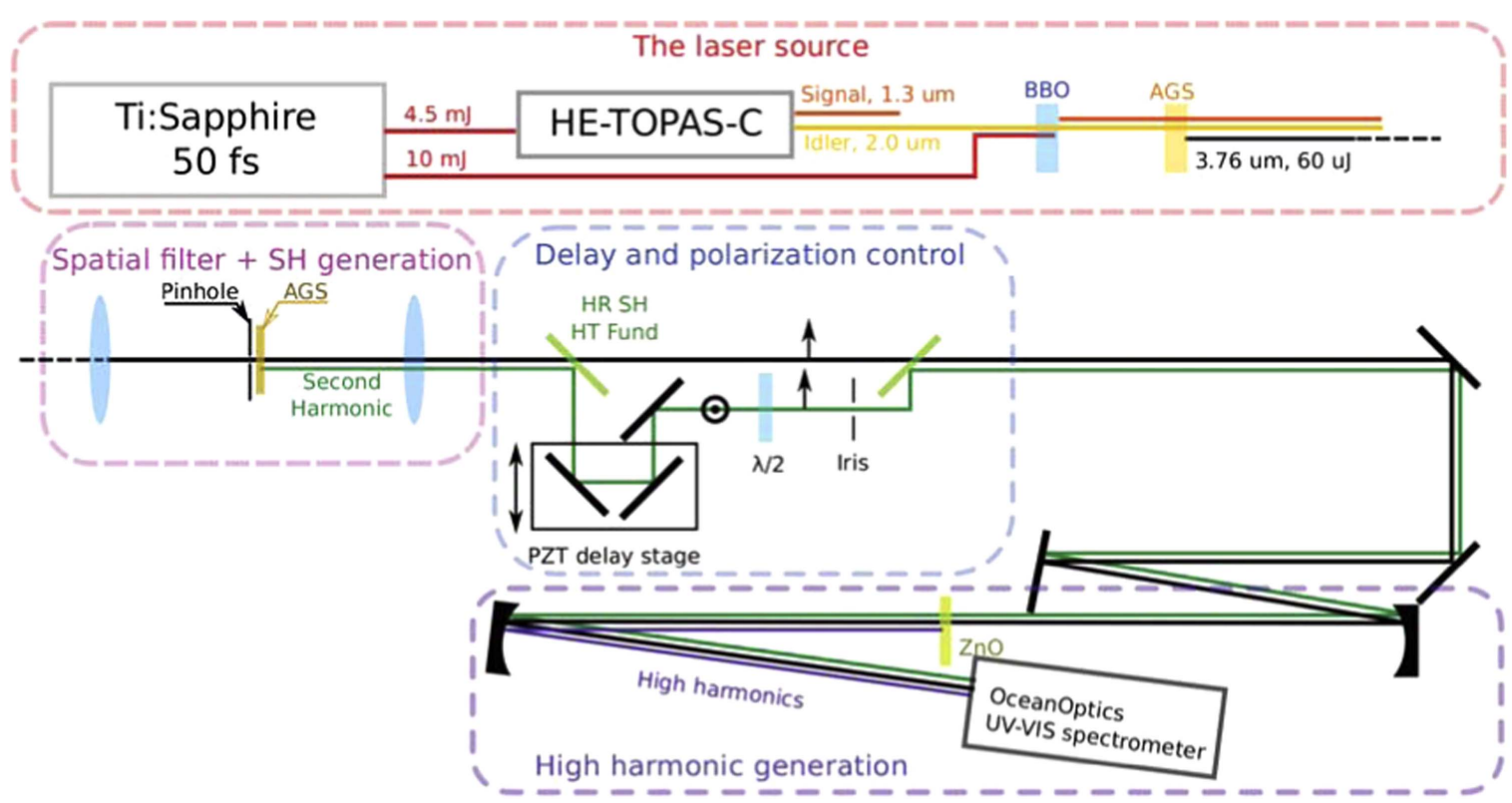

Figure 13. Sketch of the experimental setup.

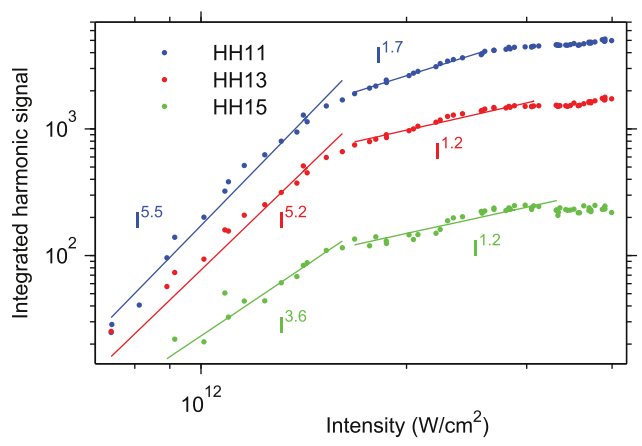

Figure 14. High harmonic signal integrated over the spectral width as a function of the fundamental intensity. For this particular experiment, the sample is a $500 \mu \mathrm{m}$ thick single $\mathrm{ZnO}$ crystal. The scaling is non-perturbative and resembles the one reported in the literature [14].

The broken symmetry results in the appearance of even harmonics. The recorded spectrum, as a function of delay between the two colours, is shown in figure 15 , for a second harmonic intensity of $9 \times 10^{-6}$ times that of the fundamental. The extremely weak second harmonic field does not significantly modify the spectrum of the odd harmonics that would be obtained in the fundamental field alone. Therefore, it is not reported here.

4.1.2. The method. The method is adapted from atomic experiments [44-46], where variations of it have allowed measuring the symmetry of atomic wavefunctions [47] and tapping into the tunnelling process [20,48].

To understand the origin of the even harmonics, it is useful to think of HHG as a collection of interferometers, each interferometer associated to emission of a specific harmonic photon energy, and in which each arm of the interferometer corresponds to trajectories of electron-hole pairs launched at subsequent laser half-cycles (see figure 16). The length of

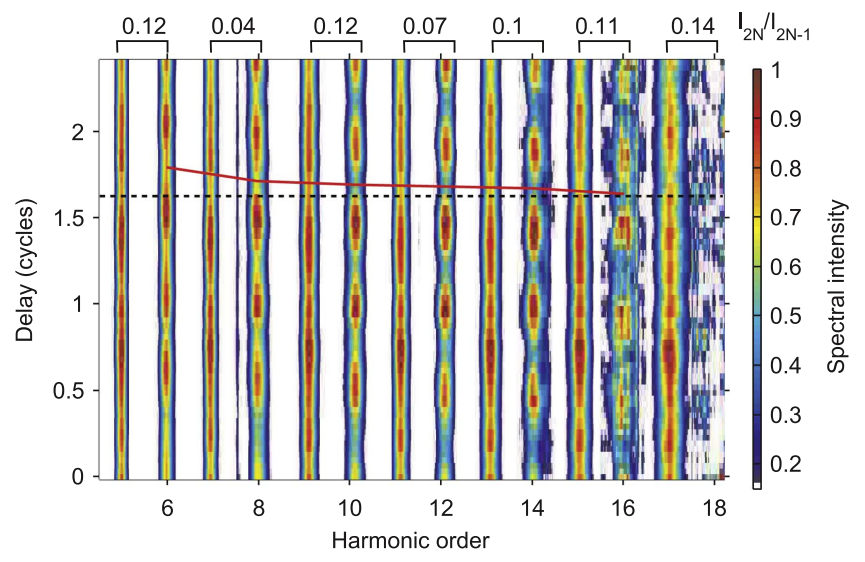

Figure 15. Measured high harmonic spectra versus the delay between the two colours (in cycles of the second harmonic); the spectral intensity is colour-coded (key at right). The even harmonic intensity modulates every half-cycle. The odd harmonics also modulate, but the modulation is extremely weak. In fact, their intensity is almost unaffected by the second harmonic field. The phase of the even harmonic modulation depends on the harmonic order, as evidenced by the red solid line relative to the black reference line (the red line links the minima in the modulation of the even harmonics). Each harmonic order is separately normalized. The relative intensity between even-order and adjacent odd-order harmonics is given at the top $\left(I_{2 N} / I_{2 N-1}\right)$. Adapted from [43].

each arm is determined by the phase of the dipole, $\exp [\mathrm{i} S]$, where $S$ is the semiclassical action accumulated between the time of birth and of recollision:

$$
S\left(\mathbf{k}, t^{\prime}, t\right)=\int_{t^{\prime}}^{t} \varepsilon_{\mathrm{g}}\left(\boldsymbol{\kappa}_{\tau}\right) \mathrm{d} \tau
$$

with $\boldsymbol{\kappa}_{\tau}=\mathbf{k}+\mathbf{A}(\tau)-\mathbf{A}(t)$.

In the fundamental field alone, each interferometer is balanced: the length of the two arms is equal but oppositely phased, corresponding to electrons (holes) travelling equal distance to the left (right) in the first half-cycle and to the right 

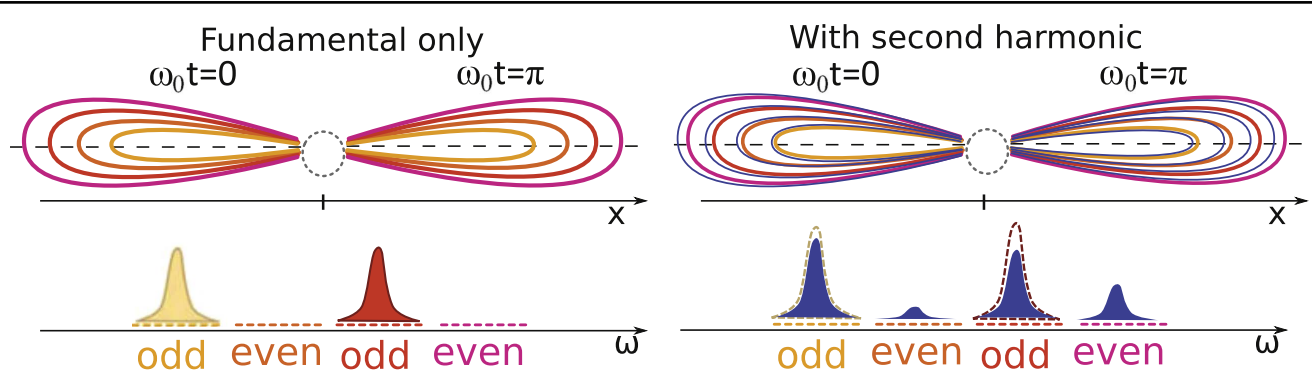

Figure 16. The (discrete) harmonic structure arises from temporal interference of emission from each atom at each laser half-cycle. Each interferometer is associated to a specific harmonic order. Each arm of the interferometer corresponds to propagation in two successive laser half-cycles. (Top left) In the fundamental field alone, electrons propagate equal distance to the left and to the right of the origin (marked by the hollow grey dashed circle) in two subsequent half-cycles. Four trajectories are depicted, colour coded to the harmonic photon energy emitted upon recollision. Propagation in each half-cycle corresponds to an arm of an interferometer. (Bottom left) Only interferometers associated to odd harmonics result in constructive interference at the output. (Top right) A properly phased second harmonic field stretches trajectories in the first half cycle, while it shrinks them in the next half cycle (blue lines). (Bottom right) In this case the interferometer is unbalanced. This results in partially constructive interference of the even harmonics. The higher the even harmonic signal, the more the phase difference between the two arms.

(left) in the next half-cycle (see top-left part of figure 16). Mathematically, the dipole moment $\mathrm{d}(t)=-\mathrm{d}\left(t+\pi / \omega_{0}\right)$. The total dipole oscillating at the frequency $n \omega_{0}$ is the coherent addition of the two arms:

$$
\begin{aligned}
p(t) & =\mathrm{d}(t) \mathrm{e}^{\mathrm{i} n \omega_{0} t}+\mathrm{d}\left(t+\pi / \omega_{0}\right) \mathrm{e}^{\mathrm{i} n \omega_{0}\left(t+\pi / \omega_{0}\right)} \\
& =\mathrm{d}(t) \mathrm{e}^{\mathrm{i} n \omega_{0} t}\left[1-\mathrm{e}^{\mathrm{i} n \pi}\right] .
\end{aligned}
$$

Clearly, the two arms constructively interfere only for interferometers associated to emission at the odd harmonics ( $n=$ odd), as illustrated in the bottom-left part of figure 16 .

A second harmonic field properly phased to the fundamental breaks the symmetry between successive half cycles. When the second harmonic adds to the fundamental field in the first half-cycle it brings electrons a little further from their holes, while they are brought a little closer in the next half-cycle, when fundamental and second harmonic subtract (see top-right part of figure 16). In optical language, the interferometers are unbalanced: phase is added to one arm while it is removed from the other. The total phase in each arm is:

$$
S_{2} \simeq S_{1}+\sigma(t, \phi)=S_{1}+\int_{t^{\prime}}^{t} \Delta \mathbf{v}\left(\boldsymbol{\kappa}_{\tau}\right) \mathbf{A}_{2}(\tau, \phi) \mathrm{d} \tau,
$$

where $S_{1}$ is the phase accumulated in the fundamental field alone (equation (31) with $\mathbf{A}(t)$ equal to the vector potential of the fundamental field), $\mathbf{A}_{2}(\tau, \phi)=\mathbf{A}_{2} \cos \left(2 \omega_{0} \tau+\phi\right)$ is the vector potential of the second harmonic and $\Delta \mathbf{v}(\mathbf{k})=\nabla_{\mathbf{k}} \varepsilon_{g}(\mathbf{k})$ the difference of the velocities of electrons and holes. The above formula can be derived by expanding equation (31) for small $\mathbf{A}_{2}$. Because $\sigma(t, \phi)=-\sigma\left(t+\pi / \omega_{0}, \phi\right)$, the total phase difference between the two arms amounts to $2 \sigma$. The uneven length of the arms of the interferometers modifies the interference as follows:

$$
\begin{aligned}
p(t) & =d_{1}(t) \mathrm{e}^{\mathrm{i} n \omega_{0} t}\left[\mathrm{e}^{\mathrm{i} \sigma(t, \phi)}-\mathrm{e}^{-\mathrm{i} \sigma(t, \phi)} \mathrm{e}^{\mathrm{i} n \pi}\right] \\
& = \begin{cases}2 d_{1}(t) \mathrm{e}^{\mathrm{i} n \omega_{0} t} \cos (\sigma) & \text { if } n \text { is odd } \\
2 \mathrm{i}_{1}(t) \mathrm{e}^{\mathrm{i} n \omega_{0} t} \sin (\sigma) & \text { if } n \text { is even }\end{cases}
\end{aligned}
$$

where $d_{1}$ is the dipole due to the fundamental field alone. Even harmonics are now generated. Their intensity is
$I_{2 N} \propto|\sin (\sigma)|^{2}$, as illustrated in the bottom-right part of figure 16. In the experiment, the intensity of the even harmonics is about $10 \%$ of the nearest odd harmonic. Therefore, one can estimate that $\sigma \simeq 0.32 \mathrm{rad}$.

In optics, the interference can be continuously modified by moving one arm of the interferometer with respect to the other. By varying the delay between fundamental and second harmonic, the asymmetry between successive half cycles felt by the electrons can be continuously adjusted. As a result the even harmonic intensity modulates with a period of half of the second harmonic cycle (see figure 15). Maximum even harmonic intensity is reached at an optimum phase $\Phi_{\mathrm{osc}}$, where the asymmetry is strongest. The red line in figure 15 links $\Phi_{\text {osc }}$ between all even harmonic orders. Because different trajectories are born and recollide at different times, each feels the second harmonic field differently. Therefore $\Phi_{\text {osc }}$ differs between harmonic orders (as evidenced by comparison with the flat dashed black line in figure 15). This behaviour is observed in gases as well.

4.1.3. The findings. To compare $\Phi_{\text {osc }}$ with theoretical predictions, the experiment is simulated using the two-band model introduced in section 3, for a second harmonic field strength of $6 \times 10^{-4}$ relative to the fundamental. This ensures that the even harmonics are $\sim 5 \%$ of the odd ones, similar to the experiment $\left(\Phi_{\mathrm{osc}}\right.$ is rather insensitive to the strength of the perturbing field, as long as the even harmonics remain 'small'). The simulations cannot account for spatial averaging over the gaussian beam profile, which can cause loss of contrast in the modulation and variations in $\Phi_{\mathrm{osc}}$. Spatial averaging can be easily avoided in the experiment by filtering the beam after the crystal. Temporal averaging, on the other hand, is accounted for by using the same pulse duration in the simulations than in the experiment. The theoretical values of $\Phi_{\text {osc }}$ are extracted from the simulated spectrograms and are plotted in figure 17 for the intraband (purple line) and interband (blue line) sources, and for their combined emission (gold dashed line). To position the experimental $\Phi_{\text {osc }}$ (black circles), an unmeasured constant phase is added (this constant phase can be measured by frequency doubling the fundamental in a phase-matched 


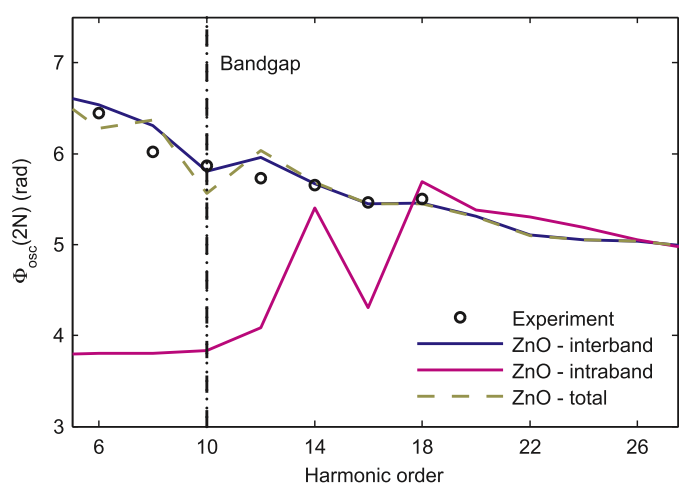

Figure 17. $\Phi_{\mathrm{osc}}(2 N)$ is extracted from figure 15 (black circles) and compared to the simulated intraband (purple line) and interband (blue line) phase. The simulated phase for their combined emission (interband plus intraband, dashed yellow line) agrees with interband emission, in agreement with interband harmonics being significantly stronger than intraband ones (figure 7). The vertical dash-dotted black line marks the minimum band gap of $\mathrm{ZnO}$, at $3.3 \mathrm{eV}$. Adapted from [43].

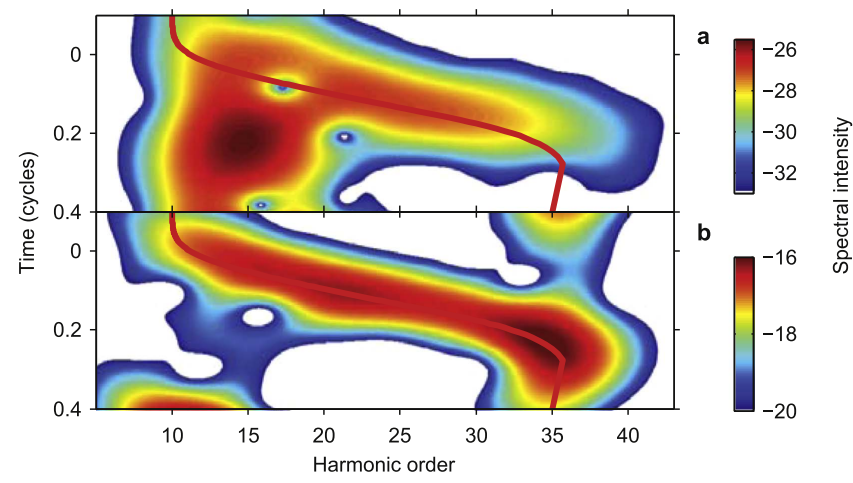

Figure 18. Time frequency analysis of the simulated experiment. Intraband emission (a) is timed to the recolliding electron-hole pairs (red line) only for harmonic orders above the 18th. Interband harmonics (b), instead, arise all from the recollision mechanism. The spectral intensity, colour-coded, is in logarithmic scale. Adapted from [43].

crystal, and beating the newly generated second harmonic field with the one used to perturb the harmonics). We find that the experiment is consistent with the theoretical prediction that interband harmonics dominate in $\mathrm{ZnO}$ for the field strength used (see figure 7).

The optimum phase of intraband harmonics above the 18th order follows that of interband harmonics, suggesting that intraband recollisions-a mechanism presented in section 3-dominates intraband emission in this harmonic range. This is further proved in figure 18, that shows the windowed Fourier transform of inter- and intraband currents (colour coded) superposed to the time of emission expected from the semiclassical model of recollisions (red lines). The time of emission of intraband harmonics (panel (a)) agrees with recollisions for harmonic orders above the 18th. Below the 18th order, the emission becomes independent on the order, suggesting that other intraband emission mechanisms take over. As a result, $\Phi_{\text {osc }}$ also becomes independent on the harmonic order in this energy range. To summarize, varation of $\Phi_{\mathrm{osc}}$ with harmonic order tags recolliding electron-hole pairs, thereby establishing a link between atomic and solid harmonics.

4.1.4. Tightening the link. This link can be strengthened further. In figure 15, the odd harmonics also modulate, but their modulation is much weaker than that of the even harmonics. This is because the added phase $\sigma$ is small enough that $I_{2 N+1} \propto|\cos (\sigma)|^{2} \simeq 1$. When the second harmonic intensity is increased to $\sim 10^{-4}$ of the fundamental (see figure 19(a)), the odd harmonics strongly modulate out of phase with the even harmonics. The added phase in each arm of the interferometer is now $\sigma \simeq \pi / 2$, because for the appropriate delay only even harmonics are generated. This regime is also observed in atomic HHG.

For even higher second harmonic field strength (figure 19(b)), the emission is substantially modified. In the highly asymmetric sum field, tunnelling only occurs once per laser cycle. This leads to simultaneous emission (or suppression) of even and odd order harmonics - a behaviour wellstudied for atomic gases [44, 45]. In the interferometer picture, the intensity - not only the path length-in each arm of the interferometer is modified. The harmonic structure arises from interference of emission at each laser cycle:

$$
\begin{aligned}
p(t) & =\mathrm{d}(t) \mathrm{e}^{\mathrm{i} n \omega_{0} t}+\mathrm{d}\left(t+2 \pi / \omega_{0}\right) \mathrm{e}^{\mathrm{i} n \omega_{0}\left(t+2 \pi / \omega_{0}\right)} \\
& =\mathrm{d}(t) \mathrm{e}^{\mathrm{i} n \omega_{0} t}\left[1+\mathrm{e}^{\mathrm{i} 2 n \pi}\right],
\end{aligned}
$$

which is always non-zero for $n=\{$ even, odd $\}$.

The band structure of the material has a strong influence on the high harmonics. This is analysed in the following section.

\subsection{Role of the band structure}

In the $\mathrm{ZnO}$ experiment, even harmonics are detected for second harmonic intensities only $10^{-6}$ of the fundamental one. This relative intensity is three orders-of-magnitude smaller than in gas phase experiments performed with $800 \mathrm{~nm}$ laser pulses. As shown in figure 20(a), electron-hole pairs in $\mathrm{ZnO}$ (black line), at the laser frequency and intensity of the experiment, accumulate four to ten times the phase they accumulate in vacuum (red line) in typical gas phase experiments with $I_{0}=10^{14} \mathrm{~W} \mathrm{~cm}^{-2}$ and $\lambda=0.8 \mu \mathrm{m}$. In the optics analogy, the arms of the interferometers are longer in solid experiments. Therefore, much weaker fields are required to unbalance the interferometers by the same phase shift.

The longer wavelength used in experiments in solids is only part of the reason why the accumulated phase is higher. The biggest contribution comes from the smaller effective mass of electrons and holes in solids as opposed to in vacuum. The effective mass is defined by the curvature of the bands:

$$
\left[m^{*}\right]^{-1}=\frac{1}{\hbar^{2}} \nabla_{k}^{2} E_{m}(k)
$$

Because the phase is determined by the band gap $\varepsilon_{g}$, it is the reduced effective mass of the electron-hole pair that comes 

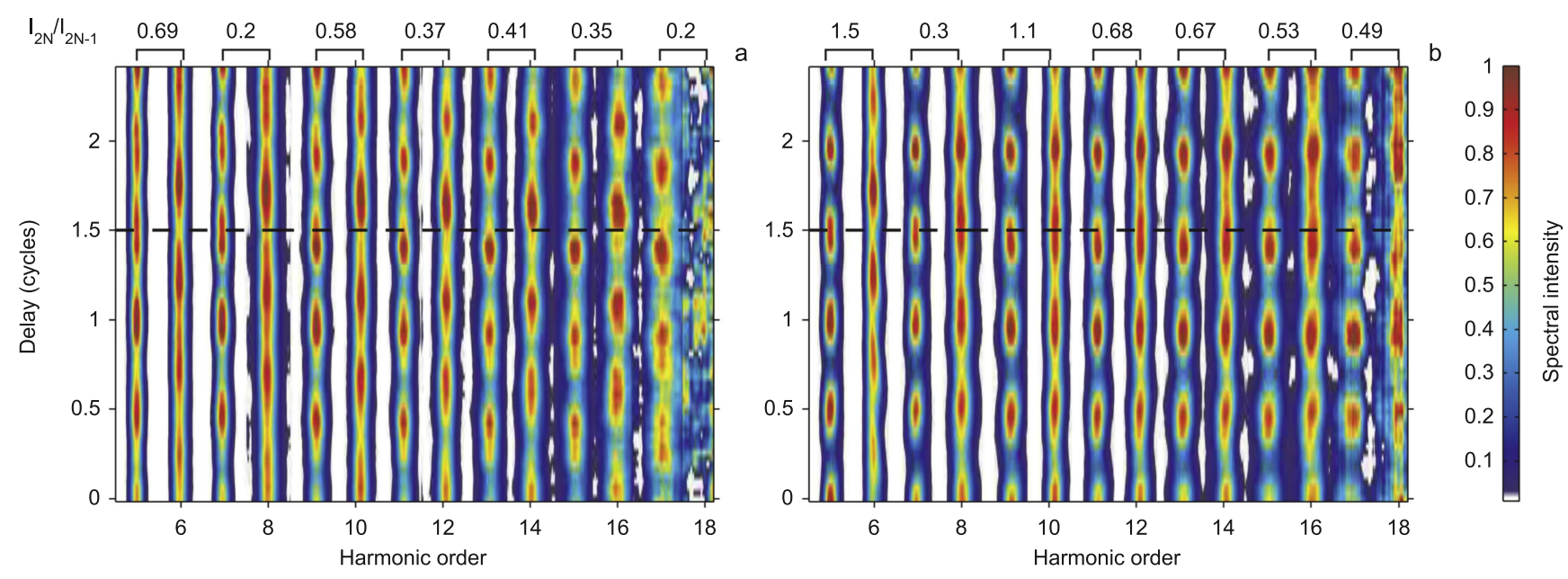

Figure 19. Experimental spectrograms for stronger second harmonic fields. (a) When the intensity of the second harmonic is increased to $1 \times 10^{-4}$ times that of the fundamental, the even and odd harmonics modulate out of phase. (b) Further increasing the intensity to $3 \times 10^{-3}$ times that of the fundamental, we find that all harmonics modulate in phase. Each harmonic order is separately normalized. The relative intensity between even-order and adjacent odd-order harmonics is given at the top. Adapted from [43].
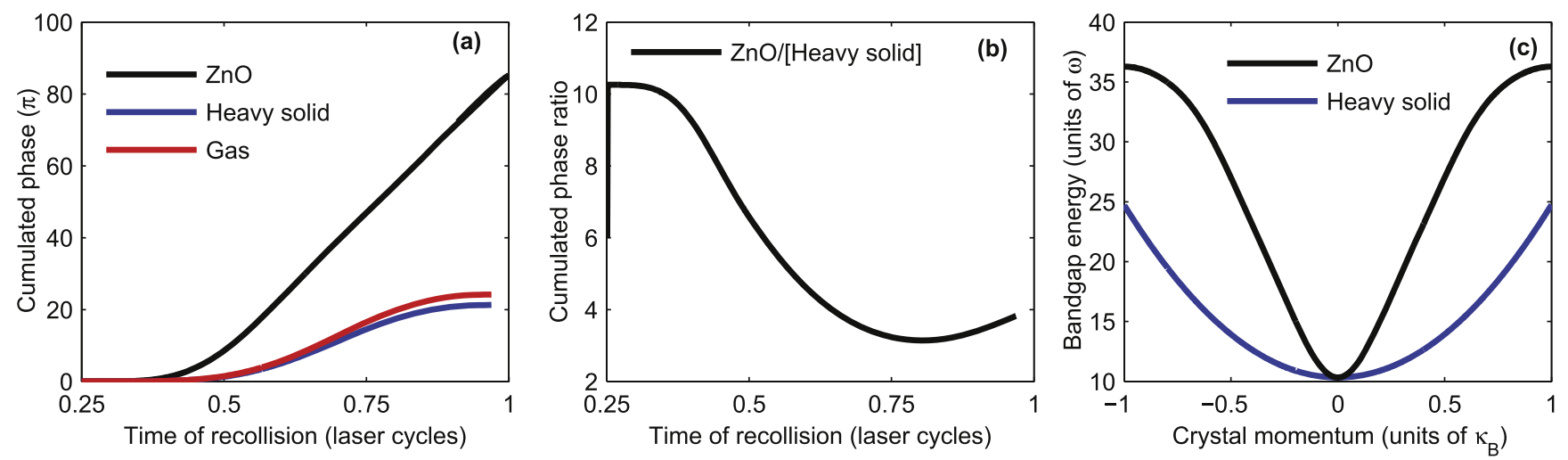

Figure 20. (a) Dipole phase (in units of $\pi$ ) accumulated by electron-hole pairs between their time of creation and recollision, as a function of the latter, calculated with equation (31). The laser parameters are $\lambda=3.76 \mu \mathrm{m}$ and $F_{0}=0.25 \mathrm{~V} \AA^{-1}$ for $\mathrm{ZnO}$ (black line) and for a solid with unitary reduced effective mass (blue line). The dipole phase accumulated in typical experiments in atomic gases with $\lambda=0.8 \mu \mathrm{m}$ and $I_{0}=10^{14} \mathrm{~W} \mathrm{~cm}^{-2}$ is plotted with a red line. (b) Ratio between the phases accumulated in $\mathrm{ZnO}$ and by electron-hole pairs having unitary reduced effective mass (blue line of panel (a)). (c) Momentum-dependent band gap of $\mathrm{ZnO}$ (black line) and of the model solid with unitary reduced effective mass (blue line).

into play:

$$
\frac{1}{m_{\mathrm{red}}^{*}}=\frac{1}{m_{c}^{*}}-\frac{1}{m_{v}^{*}}=\frac{1}{\hbar^{2}} \nabla_{k}^{2} \varepsilon_{g}(k) .
$$

The blue line in figure 20(a) is the phase accumulated by electron-hole pairs having unitary mass $\left(m_{\mathrm{red}}^{*}=1\right)$ in a solid under the conditions of the $\mathrm{ZnO}$ experiment. The band gap used is plotted in blue in panel (c), and is compared to the band gap of $\mathrm{ZnO}$ (black line). The ratio between the black and blue phases is plotted in panel (b). In $\mathrm{ZnO}$, the reduced effective mass calculated with the band gap of figure 20(c) is $m_{\text {red }}^{*}=0.1 m_{e}$. Therefore, it explains why the accumulated phase for the short trajectories, which explore the band gap only around the minimum, is $\sim 10$ times that in vacuum (for which $m_{\text {red }}^{*}=m_{e}$ ). Longer trajectories explore regions of the reciprocal space for which the quadratic approximation, equation (35), does not hold. Therefore, the ratio in the accumulated phase between $\mathrm{ZnO}$ and vacuum differs from 10 .
In conclusion, it is the different band structure of $\mathrm{ZnO}$ and vacuum, the integrated quantity in equation (31), that allows the greater sensitivity of $\mathrm{ZnO}$ to perturbing fields at fundamental intensities which are $\sim 100$ times weaker than in gas phase experiments.

In $\mathrm{ZnO}$, a clear spectral signature is imposed on the high harmonic beam using a control beam having a peak electric field of only $\sim 5 \mathrm{~V} \mu \mathrm{m}^{-1}$. Fields of this magnitude are present in electronic circuits. Therefore, if the same sensitivity is found in silicon, it seems possible to record movies of working electrical circuits. When the circuit is off, harmonics generated from the spatially complex circuit will diffract according to the shape of the structures. When the circuit is on, the diffraction is modified by the instantaneous distribution of the internal fields. Comparison of the 'off' with the 'on' pattern will return a snapshot of the working circuit. Furthermore, using solids as platforms for HHG allows to merge attosecond physics with nonlinear optics and 
plasmonics. It will be possible, for example, to sense the evolution of plasmonic fields in space as well as in time.

\subsection{Chirp of high harmonic pulses}

One of the most important applications of atomic HHG is the possibility to synthesize XUV laser pulses with a duration of a fraction of a cycle of the fundamental; that is, several tens of attoseconds [7]. In solids, sub-cycle bursts of harmonic radiation are also created.

How short such bursts can be? Short pulses require wide bandwidths and nearly flat spectral phases (small chirps). The band structure of $\mathrm{ZnO}$ allows generation of 20 harmonic orders at the conditions of the experiment, corresponding to $7.6 \mathrm{eV}$ bandwidth. A transform-limited gaussian spectrum with half of this bandwidth corresponds to high harmonic pulses with a duration of 480 as (FWHM). However, analogously to the atomic case, the generation process gives an intrinsic temporal chirp to the harmonics that prevents the generation of transform limited pulses. The chirp arises from the variation in the emission time of different harmonic orders [49, chapter 4]:

$$
C=\frac{\mathrm{d} t}{\mathrm{~d}(n \omega)}=(2 \hbar b)^{-1},
$$

with harmonic order $n(t)$ a function of the return (emission) time. The parameter $b$ is defined in the time-dependent electric field:

$$
E(t)=E_{0} \mathrm{e}^{-a t^{2}} \mathrm{e}^{-\mathrm{i} b t^{2}},
$$

where the pulse duration $\tau=\sqrt{2 \ln (2)} / a$. The Fourier transform of equation (37) is:

$$
E(\omega)=E_{0} \mathrm{e}^{-\frac{a \omega^{2}}{4\left(a^{2}+b^{2}\right)}} \mathrm{e}^{-\mathrm{i} \frac{b \omega^{2}}{4\left(a^{2}+b^{2}\right)}}=E_{0} \mathrm{e}^{-2 \ln (2) \frac{\omega^{2}}{\Delta \omega^{2}}} \mathrm{e}^{-\frac{1}{2} \operatorname{GDD} \omega^{2}} .
$$

By solving the equality, one finds that the pulse duration is:

$$
\tau=\frac{\sqrt{2} \ln (2)}{|b| \tau_{t l}} \sqrt{1+\sqrt{1-\frac{b^{2} \tau_{t l}^{4}}{\ln (2)^{2}}}} \simeq \frac{2 \ln (2)}{|b| \tau_{t l}}=\Delta \omega \hbar C,
$$

and the GDD is:

$$
\mathrm{GDD} \simeq \frac{1}{2 b\left(1+\frac{b^{2} \tau_{t l}^{4}}{(2 \ln (2))^{2}}\right)} \simeq \hbar C .
$$

Figure 21(a) shows the variation of harmonic order with time (solid blue line) obtained from a theoretical calculation performed for laser frequency $\omega_{0}=0.014$ a.u. and $F_{0}=0.0046$ a.u. The first derivative (the chirp) calculated at half of the maximum band gap (at $7.5 \mathrm{eV}$ ) for the long and short trajectories is also plotted (dashed blue lines). It amounts to $-132 \mathrm{as} \mathrm{eV}^{-1}$ and $+288 \mathrm{as} \mathrm{eV}^{-1}$ for the long and short trajectories respectively. Equation (40) predicts a GDD

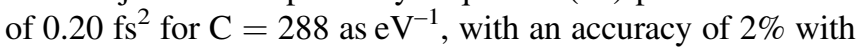
respect to that predicted by the full equation (38). The pulse duration for the whole bandwidth is $2.2 \mathrm{fs}$.
The pulse can be shortened either by propagating it through a material with opposite GDD (in which case the pulse becomes transform limited), or by reducing the bandwidth. In the presence of GDD, a transform limited Gaussian pulse of duration $\tau_{t l}$ (FWHM) is broadened to a duration [49, chapter 1] of,

$$
\tau_{c}=\tau_{t l} \sqrt{1+\frac{(4 \ln 2)^{2} \mathrm{GDD}^{2}}{\tau_{t l}^{4}}} .
$$

The shorter the pulse and the wider the spectrum, the more severe it is affected by the chirp. As a result, as long as the chirp cannot be compensated, there exists an optimum bandwidth that generates the shortest pulse. The duration of the high-harmonic pulse as a function of its bandwidth is shown in figure 21(b). The shortest possible pulse with the given chirp is achieved for $2.5 \mathrm{eV}$ bandwidth and lasts $1.02 \mathrm{fs}$ (transform limit is $0.73 \mathrm{fs}$ ).

So far, the estimate on the achievable pulse duration has not taken into account how the harmonic intensity changes with harmonic order, a factor that determines the actual bandwidth achievable in experiments. In fact, a fast drop of harmonic intensity with order has been measured from $\mathrm{ZnO}$ [14]. This suggests that multi-eV bandwidths are hard to achieve in $\mathrm{ZnO}$, however a $\sim 2.5 \mathrm{eV}$ FWHM seems possible. It corresponds to the optimum bandwidth in figure 21(b). Therefore, by optimizing material parameters and by going to wider band gap materials, sub-fs high-harmonic pulses from solids will be achieved. Wider bandwidths can also be obtained by excitation to higher lying conduction bands $[32,50]$ or lower lying valence bands.

The high harmonic pulses propagate inside the generating crystal, so that linear material dispersion changes the pulses that reach the exit surface. In practice, only above band gap harmonics that are generated within one absorption length of the material are measured [51]. Because absorption lengths are on the order of few tens of nanometers, it is unlikely that linear dispersion plays any role.

In the experiment in $\mathrm{ZnO}$ [43], a harmonic chirp of 380 as $/ \mathrm{eV}$ at the 16th harmonic order can be extracted by comparing $\Phi_{\mathrm{osc}}(2 N)$ of figure 17 to that calculated with the semiclassical model (red line of figure 18). It corresponds to a train of pulses with duration of $1.7 \mathrm{fs}$ (before dispersion compensation) for a $\sim 2 \mathrm{eV}$ bandwidth. A more accurate determination of the chirp must take into account the complex saddle points [34, 46]. Finally, the recombination dipole phase-which is part of the harmonic chirp-is not imprinted in the 2-colour spectrogram.

4.3.1. Comparison with gases. Chirps obtained from solids at the conditions of the experiment are larger than those obtained in gases, which typically are $\sim 10 \mathrm{as} \mathrm{eV}^{-1}$. Small chirp in gases is attributed to the two orders of magnitude higher intensity typically used in experiments, which in turn results in wider bandwidth of the harmonic spectrum and smaller shifts of emission time per harmonic order.

Besides the laser parameters, the band structure also affects the chirp. Figure 21(a) compares the chirp from $\mathrm{ZnO}$ 

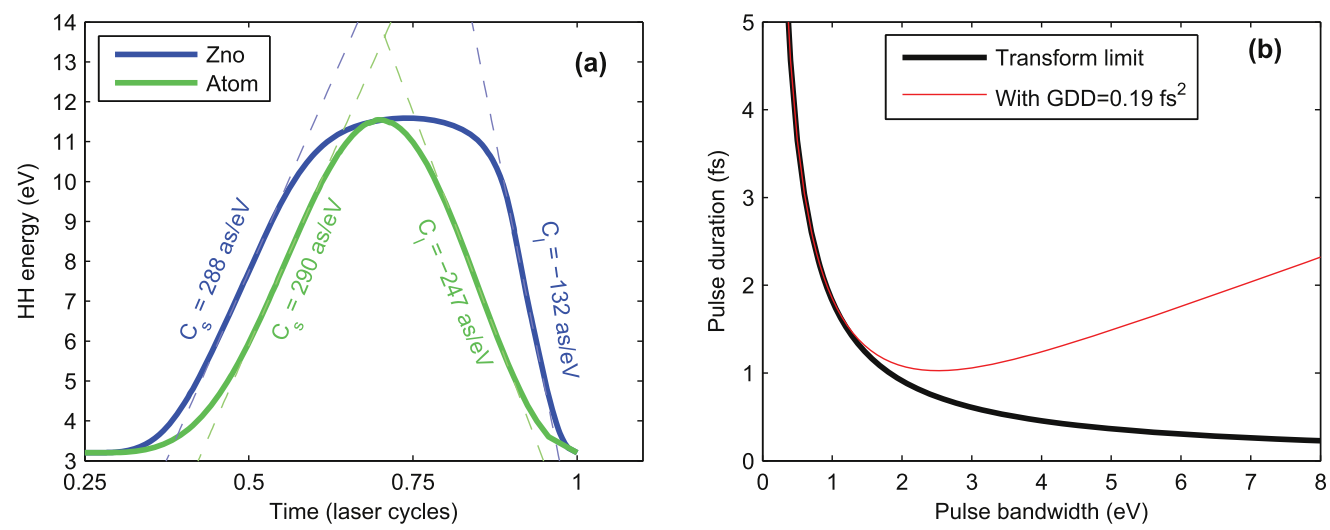

Figure 21. (a) High harmonic photon energy as a function of the emission time for $\mathrm{ZnO}$ at $F_{0}=0.0046$ a.u. (blue solid line) and for an atomic system with the same band gap as $\mathrm{ZnO}$ at $F_{0}=0.0087$ a.u. (green solid line). The latter field strength is chosen to equate the cut-off. The atomic ionization potential is set equal to the minimum bandgap $E_{\mathrm{g}}$. Laser frequency is $\omega_{0}=0.0141$ a.u. The slope of the curves determines the chirp of the high harmonic pulses. The dashed lines are the derivatives taken at $7.5 \mathrm{eV}$ for the long $\left(C_{l}\right)$ and short $\left(C_{s}\right)$ trajectories. The values of the chirps are reported along the lines. (b) Variation of the pulse duration as a function of the pulse bandwidth. The short trajectory chirp equivalent to GDD $=0.19 \mathrm{fs}^{2}$ broadens the pulse (red line) with respect to its transform limit (black line). For this GDD the shortest pulse (1.02 fs) is obtained for a $2.5 \mathrm{eV}$ bandwidth. (C) 2015 IEEE. Adapted, with permission, from [25].

and from atomic gases for field strengths that yield the same cut-off harmonic (at the same fundamental frequency). The atomic emission times and chirps are the green solid and dashed curves respectively. Whereas the atomic chirp of the short trajectory is $290 \mathrm{as} \mathrm{eV}^{-1}$ (comparable to the one from $\mathrm{ZnO}$ ), the chirp of the long trajectory is -247 as eV$^{-1}$, almost twice the one from $\mathrm{ZnO}$.

\section{Recent trends}

Most investigations to date focused on understanding the mechanism responsible for high harmonic emission.

Two groups have reported emission associated to Bloch or Velocity harmonics. In the first case [16], a GaSe crystal ( $2.1 \mathrm{eV}$ bandgap) was excited by pulses with $10 \mu \mathrm{m}$ central wavelength. On the basis of the agreement between the simulated and experimental spectra, the authors concluded that electron-hole pairs are created by interband excitation and that harmonics are radiated as a result of acceleration of electrons and holes in their respective bands. In a followup experiment [41], the same group has demonstrated that the emitted harmonics are all phased together-a finding that strengthens their interpretation. Experiments performed in $\mathrm{SiO}_{2}(\sim 9$ eV bandgap) at a much shorter wavelength $(\sim 800$ $\mathrm{nm}$ ) also demonstrated emission of chirp-free harmonics [52]. In this experiment, the authors measured harmonics above the maximum bandgap between the two closest valence and conduction bans. In this situation, the recollision contribution could be indeed suppressed.

Crystals that lack inversion symmetry, when appropriately oriented with respect to the laser polarization, can generate second harmonic. Because the lack of symmetry is a general property of the material, these crystals are also expected to generate even harmonics. Their origin, however, cannot be interpreted in terms of the perturbative nonlinear susceptibility tensor. The experiments performed in GaSe
$[16,41]$ show even harmonics that are as bright as the odd harmonics. This situation suggests a very strong symmetry breaking whereby harmonic emission happens only once per laser cycle. The authors suggest that ionization is quenched every other laser half-cycle by the interference of two quantum-paths. One path is a direct excitation from the topmost valence band directly to the first conduction band. In the other path the electron starts from the topmost valence band, is temporarily excited to the valence band right below from where it is ultimately excited to the conduction band. The transition between the two valence bands, which is initially blocked by Pauli exclusion, opens up as soon as electrons are directly excited from the lower valence band to the conduction band.

In an alternative experiment performed on $\mathrm{MoS}_{2}$ (a 2D material) [53], the authors ascribe the even harmonics to the Berry curvature of the bands. The Berry curvature acts as a pseudo-Lorentz force on the electron, and as a result the even harmonics are polarized perpendicularly to the odd harmonics. Indeed, most of the even harmonic intensity is found in the perpendicular polarization. In the same experiment, the authors argue that the 2D nature of the material enhances high harmonic emission due to the stronger electron-hole interaction in the monolayer.

\section{Outlook}

In our opinion, HHG from colliding electron-hole pairs offers an advantage that is absent in non-recollision based mechanisms: the encoding of well defined trajectories in the spectral phase of the high harmonics. This property is commonly used in high harmonic spectroscopies of atoms and molecules. Recollision based HHG in solids provides the opportunity to directly extend these methods to the solid phase. Not only field-free solids can be probed, but-what is new-also when they are dressed by a strong laser field. High 

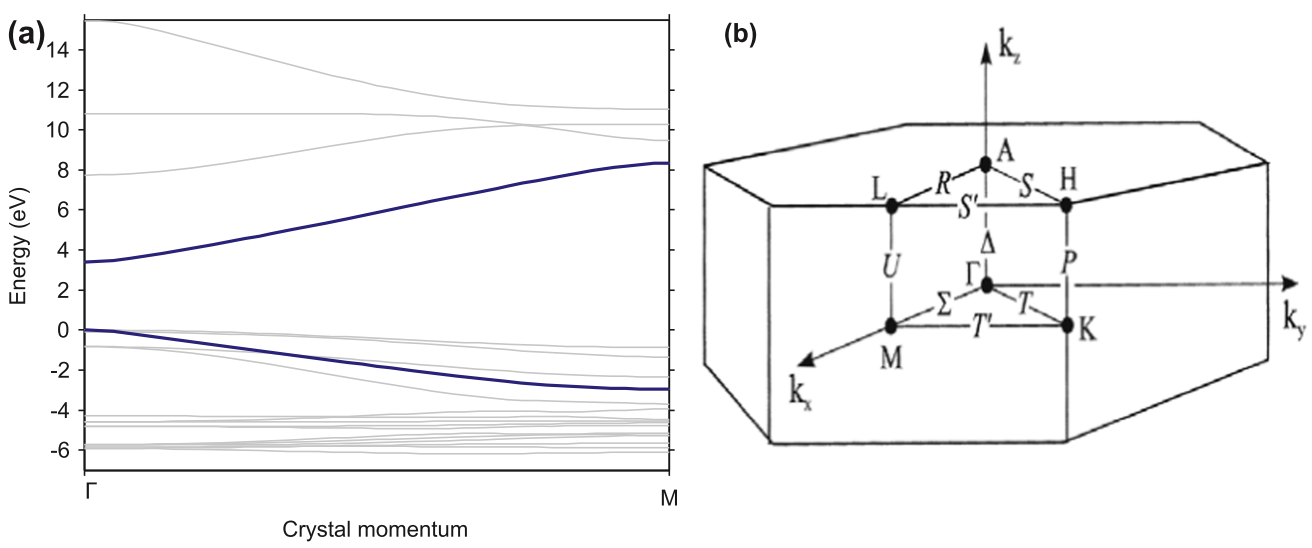

Figure 22. (a) Band structure of $\mathrm{ZnO}$ along the $\Gamma-M$ direction of the Brillouin zone, calculated with LDA-ABINIT. The split-off valence and first conduction bands of $\mathrm{ZnO}$ are coloured blue. (b) Brillouin zone of wurtzite $\mathrm{ZnO}$. The high symmetry points are indicated.

harmonics can help settle some long standing debates about ionization mechanisms in dielectrics versus semiconductors $[10,11]$, the origin of laser induced damage and, possibly, how to control it.

Solid materials exhibit a rich phenomenology not accessible by gases: they can exhibit strong electron-electron correlations and change properties under high pressures. Solids can be more easily manipulated than molecules: crystals are readily aligned and permanent electric and magnetic dipoles can be switched at will in ferroelectric and ferromagnetic materials. Furthermore, crystals allow to study the influence of order on HHG, be it magnetic (for example antiferromagnetism versus ferromagnetism), electronic (for example charge density waves), or atomic (for example to probe the transition from crystalline to amorphous). According to this view, solids may even be better platforms to perform many experiments originally proposed for the gas phase. Moreover, solids can have sharp boundaries. The topology of heterostructures, such as junctions, quantum wells, and the like, can all be probed by $\mathrm{HHG}$, as well as the intrinsic electric fields at the interfaces between different materials. And finally, solids can be patterned with micro and nanostructures. Nanoplasmonic devices and metamaterials are obvious examples of how nanoscale features can influence light generation and propagation. It seems almost obvious to merge these fields of research, including nonlinear optics, with HHG in solids.

Despite progress in understanding recollision-based HHG in solids, much is unknown. For example: the role of diffusion of the wavepackets, the role of excitons and screening, the role of dephasing. Dephasing, for example, disrupts the mutual coherence of electrons with their correlated holes. It is very likely that this information is encoded in the recollision-based harmonics.

\section{Appendix. Band structure of $\mathrm{ZnO}$ and details on the simulations}

The Semiconductor Bloch equations, equation (18), are integrated numerically with Runge-Kutta method. The inter- and intraband currents and their respective high-harmonic spectra are separately calculated with equation (16). The numerical value of the transition dipole moment $d_{c v}$ has been extracted from tabulated data [30] and its $k$-dependence is neglected.

The laser is characterized by field amplitude $\mathbf{F}(t)=$ $F_{0} f(t) \cos \left(\omega_{0} t\right) \hat{\mathbf{x}}$, consisting of a temporal Gaussian envelope $f(t)$ and cosine carrier with frequency $\omega_{0}$. The input electric field is the one inside the material. The vacuum field strength is obtained by taking into account the reflection loss at the airsolid interface: $F_{\mathrm{vac}}=(n+1) F_{0} / 2$, where $n$ is the refractive index of the solid.

The bands calculated with density functional theory are fitted to a Fourier series expansion which is used in the integration of equations (18). 3D and 1D simulations are performed. The 3D band structure is approximated as:

$$
E_{m}(\mathbf{k})=E_{m, x}\left(k_{x}\right)+E_{m, y}\left(k_{y}\right)+E_{m, z}\left(k_{z}\right) .
$$

For each direction in reciprocal space $(i=x, y, z)$ :

$$
\begin{gathered}
E_{v, i}\left(k_{i}\right)=\sum_{j=0}^{\infty} \alpha_{v, i}^{j} \cos \left(j k_{i} a_{i}\right) \\
E_{c, i}\left(k_{i}\right)=E_{g}+\sum_{j=0}^{\infty} \alpha_{c, i}^{j} \cos \left(j k_{i} a_{i}\right)
\end{gathered}
$$

with $a_{i}=2 \pi / \kappa_{i}$ where $\kappa_{i}$ the reciprocal space wavevector along coordinate $i$. Only one valence and one conduction bands are considered, unless when mentioned otherwise. Initially, the valence band is completely full, and the conduction band completely empty.

Parameters for $\mathrm{ZnO}$. $\mathrm{ZnO}$ has a wurtzite structure at ambient conditions. The Brillouin zone of $\mathrm{ZnO}$ is depicted in figure 22(b). The energy bands along some high-symmetry directions obtained by $a b$ initio calculations in the local density approximations (ABINIT), are plotted in panel (a). The split-off valence and first conduction bands are highlighted in blue.

In all simulations, the crystallographic axis have been oriented such that $\hat{\mathbf{x}}\|\Gamma-M, \hat{\mathbf{y}}\| \Gamma-K$, and $\hat{\mathbf{z}} \| \Gamma-A$ (optical axis); along these directions $\left(a_{x}, a_{y}, a_{z}\right)=(5.32,6.14,9.83)$ a.u. The expansion coefficients $\alpha_{m, i}^{j}(i=x, y, z, j=0,1 \ldots)$ 
Table 1. Coefficients of the expansion of the NL-EPM bands from [54] for the crystal field split valence band and the first conduction band.

\begin{tabular}{lrc}
\hline & Valence band & Conduction band \\
\hline$\alpha_{0, x}$ & -0.0928 & 0.0898 \\
$\alpha_{1, x}$ & 0.0705 & -0.0814 \\
$\alpha_{2, x}$ & 0.0200 & -0.0024 \\
$\alpha_{3, x}$ & -0.0012 & -0.0048 \\
$\alpha_{4, x}$ & 0.0029 & -0.0003 \\
$\alpha_{5, x}$ & 0.0006 & -0.0009 \\
$\alpha_{0, y}$ & -0.0307 & 0.1147 \\
$\alpha_{1, y}$ & 0.0307 & -0.1147 \\
$\alpha_{0, z}$ & -0.0059 & 0.0435 \\
$\alpha_{1, z}$ & 0.0059 & -0.0435 \\
\hline
\end{tabular}

for valence $(m=v)$ and conduction $(m=c)$ band are obtained from expanding the bands calculated with a NonLocal Empirical Pseudopotential Method (NL-EPM, described in [54]) up to $j=5$ for $\Gamma-M$; the nearest neighbour expansion is used for $\Gamma-K$ and $\Gamma-A$. The resulting coefficients are listed in table 1 . The bandgap energy at the $\Gamma$ point is given by $E_{\mathrm{g}}=0.1213$ a.u. $(3.3 \mathrm{eV})$. It should be noted here that both the local density approximation (LDAABINIT) and NL-EPM were used to determine the $\mathrm{ZnO}$ band structure. While some quantitative differences exist, the conclusions remain unchanged.

The transition dipole moment is $\mathbf{d}=(3.46,3.46,3.94)$ a.u.

\section{References}

[1] Boyd R W 2003 Nonlinear Optics (New York: Academic)

[2] Itatani J, Levesque J, Zeidler D, Niikura H, Pépin H, Kieffer J-C, Corkum P B and Villeneuve D M 2004 Tomographic imaging of molecular orbitals Nature 432 867-71

[3] Vozzi C, Negro M, Calegari F, Sansone G, Nisoli M, De Silvestri S and Stagira S 2011 Generalized molecular orbital tomography Nat. Phys. 7 822-6

[4] Worner H, Bertrand J, Kartashov D, Corkum P and Villeneuve D 2010 Following a chemical reaction using high-harmonic spectroscop Nature 466 604-7

[5] Smirnova O, Mairesse Y, Patchkovskii S, Dudovich N, Villeneuve D, Corkum P and Ivanov M Y 2009 High harmonic interferometry of multi-electron dynamics in molecules Nature 460 972-7

[6] Shiner A, Schmidt B, Trallero-Herrero C, Wörner H, Patchkovskii S, Corkum P, Kieffer J, Légaré F and Villeneuve D 2011 Probing collective multi-electron dynamics in xenon with high-harmonic spectroscopy Nat. Phys. 7 464-7

[7] Zhao K, Zhang Q, Chini M, Wu Y, Wang X and Chang Z 2012 Tailoring a 67 attosecond pulse through advantageous phase-mismatch Opt. Lett. 37 3891-3

[8] Goulielmakis E et al 2010 Real-time observation of valence electron motion Nature 466 739-43

[9] Wang H, Chini M, Chen S, Zhang C-H, He F, Cheng Y, Wu Y, Thumm U and Chang Z 2010 Attosecond time-resolved autoionization of argon Phys. Rev. Lett. 105143002

[10] Schultze M et al 2013 Controlling dielectrics with the electric field of light Nature $49375-8$
[11] Schultze M et al 2014 Attosecond band-gap dynamics in silicon Science 346 1348-52

[12] Neppl S et al 2015 Direct observation of electron propagation and dielectric screening on the atomic length scale Nature 517 342-6

[13] Calegari F et al 2014 Ultrafast electron dynamics in phenylalanine initiated by attosecond pulses Science 346 336-9

[14] Ghimire S, DiChiara A D, Sistrunk E, Agostini P, DiMauro L F and Reis D A 2011 Observation of high-order harmonic generation in a bulk crystal Nat. Phys. 7 $138-41$

[15] Luu T, Garg M, Kruchinin S Y, Moulet A, Hassan M T and Goulielmakis E 2015 Extreme ultraviolet high-harmonic spectroscopy of solids Nature 521 498-502

[16] Schubert O et al 2014 Sub-cycle control of terahertz highharmonic generation by dynamical bloch oscillations Nat. Photon. 8 119-23

[17] Vampa G, Hammond T J, Thiré N, Schmidt B E, Légaré F, Klug D D and Corkum P B 2016 Generation of high harmonics from silicon arXiv:1605.06345

[18] Pfeifer T, Spielmann C and Gerber G 2006 Femtosecond x-ray science Rep. Prog. Phys. 69443

[19] Corkum P B 1993 Plasma perspective on strong field multiphoton ionization Phys. Rev. Lett. 711994

[20] Shafir D, Soifer H, Bruner B D, Dagan M, Mairesse Y, Patchkovskii S, Ivanov M Y, Smirnova O and Dudovich N 2012 Resolving the time when an electron exits a tunnelling barrier Nature $485343-6$

[21] Popmintchev T et al 2012 Bright coherent ultrahigh harmonics in the kev $\mathrm{x}$-ray regime from mid-infrared femtosecond lasers Science 336 1287-91

[22] Gonze X et al 2009 Abinit: First-principles approach to material and nanosystem properties Comput. Phys. Commun. 180 2582-615

[23] Zhokhov P and Zheltikov A 2014 Field-cycle-resolved photoionization in solids Phys. Rev. Lett. 113133903

[24] Hawkins P G and Ivanov M Y 2013 Role of subcycle transition dynamics in high-order-harmonic generation in periodic structures Phys. Rev. A 87063842

[25] Vampa G, McDonald C, Fraser A and Brabec T 2015 Highharmonic generation in solids: bridging the gap between attosecond science and condensed matter physics IEEE $J$. Sel. Top. Quantum Electron. 21 1-10

[26] Blount E 1962 Formalisms of band theory Solid State Phys. 13 305-73

[27] Lewenstein M, Balcou P, Ivanov M Y, Lhuillier A and Corkum P B 1994 Theory of high-harmonic generation by low-frequency laser fields Phys. Rev. A 492117

[28] Haug H and Koch S W 1990 Quantum Theory of the Optical and Electronic Properties of Semiconductors vol 5 (Singapore: World Scientific)

[29] Golde D, Meier T and Koch S 2008 High harmonics generated in semiconductor nanostructures by the coupled dynamics of optical inter-and intraband excitations Phys. Rev. B 77075330

[30] Vampa G, McDonald C, Orlando G, Klug D, Corkum P and Brabec T 2014 Theoretical analysis of high-harmonic generation in solids Phys. Rev. Lett. 113073901

[31] McDonald C, Vampa G, Corkum P and Brabec T 2015 Interband bloch oscillation mechanism for high-harmonic generation in semiconductor crystals Phys. Rev. A 92033845

[32] Wu M, Ghimire S, Reis D A, Schafer K J and Gaarde M B 2015 High-harmonic generation from bloch electrons in solids Phys. Rev. A 91043839

[33] Keldysh L 1965 Ionization in the field of a strong electromagnetic wave Sov. Phys. JETP 20 1307-14

[34] Smirnova O and Ivanov M 2013 Multielectron high harmonic generation: simple man on a complex plane Attosecond and XUV Spectroscopy: Ultrafast Dynamics and Spectroscopy (New York: Wiley) ch 7 
[35] Vampa G, McDonald C, Orlando G, Corkum P and Brabec T 2015 Semiclassical analysis of high harmonic generation in bulk crystals Phys. Rev. B 91064302

[36] Brabec T, Ivanov M Y and Corkum P B 1996 Coulomb focusing in intense field atomic processes Phys. Rev. A 54 R2551

[37] Salieres P, L'Huillier A and Lewenstein M 1995 Coherence control of high-order harmonics Phys. Rev. Lett. 743776

[38] Becker P, Fragnito H, Cruz C B, Fork R, Cunningham J, Henry J and Shank C 1988 Femtosecond photon echoes from band-to-band transitions in gaas Phys. Rev. Lett. 611647

[39] Portella M, Bigot J-Y, Schoenlein R, Cunningham J and Shank C 1992 k-space carrier dynamics in gaas Appl. Phys. Lett. 60 2123-5

[40] Hüfner S 2013 Photoelectron Spectroscopy: Principles and Applications (Berlin: Springer)

[41] Hohenleutner M, Langer F, Schubert O, Knorr M, Huttner U, Koch S, Kira M and Huber R 2015 Real-time observation of interfering crystal electrons in high-harmonic generation Nature 523 572-5

[42] Brunel F 1990 Harmonic generation due to plasma effects in a gas undergoing multiphoton ionization in the high-intensity limit J. Opt. Soc. Am. B 7 521-6

[43] Vampa G, Hammond T, Thiré N, Schmidt B, Légaré F, McDonald C, Brabec T and Corkum P 2015 Linking high harmonics from gases and solids Nature 522 462-4

[44] Dudovich N, Smirnova O, Levesque J, Mairesse Y, Ivanov M Y, Villeneuve D and Corkum P B 2006 Measuring and controlling the birth of attosecond XUV pulses Nat. Phys. 2 781-6

[45] He X, Dahlström J, Rakowski R, Heyl C, Persson A, Mauritsson J and LHuillier A 2010 Interference effects in two-color high-order harmonic generation Phys. Rev. A 82 033410

[46] Dahlström J, L'Huillier A and Mauritsson J 2011 Quantum mechanical approach to probing the birth of attosecond pulses using a two-colour field J. Phys. B: At. Mol. Opt. Phys. 44095602

[47] Shafir D, Mairesse Y, Villeneuve D, Corkum P and Dudovich N 2009 Atomic wavefunctions probed through strong-field light-matter interaction Nat. Phys. 5 412-6

[48] Pedatzur O et al 2015 Attosecond tunnelling interferometry Nat. Phys. 11 815-9

[49] Chang Z 2011 Fundamentals of Attosecond Optics (Boca Raton, FL: CRC Press)

[50] Hawkins P G, Ivanov M Y and Yakovlev V S 2015 Effect of multiple conduction bands on high-harmonic emission from dielectrics Phys. Rev. A 91013405

[51] Ghimire S, DiChiara A D, Sistrunk E, Ndabashimiye G, Szafruga U B, Mohammad A, Agostini P, DiMauro L F and Reis D A 2012 Generation and propagation of high-order harmonics in crystals Phys. Rev. A 85043836

[52] Garg M, Zhan M, Luu T, Lakhotia H, Klostermann T, Guggenmos A and Goulielmakis E 2016 Multi-petahertz electronic metrology Nature 538 359-63

[53] Liu H, Li Y, You Y S, Ghimire S, Heinz T F and Reis D A 2017 High-harmonic generation from an atomically thin semiconductor Nat Phys. 13 262-265

[54] Goano M, Bertazzi F, Penna M and Bellotti E 2007 Electronic structure of wurtzite zno: Nonlocal pseudopotential and ab initio calculations J. Appl. Phys.

102083709 